\title{
Towards Autonomous Operation by Advanced Process Control-Process Analytical Technology for Continuous Biologics Antibody Manufacturing
}

\author{
Heribert Helgers, Axel Schmidt (D), Lara Julia Lohmann (D, Florian Lukas Vetter, Alex Juckers, Christoph Jensch, \\ Mourad Mouellef, Steffen Zobel-Roos and Jochen Strube *
}

check for

updates

Citation: Helgers, H.; Schmidt, A.; Lohmann, L.J.; Vetter, F.L.; Juckers, A.; Jensch, C.; Mouellef, M.; Zobel-Roos, S.; Strube, J. Towards Autonomous Operation by Advanced Process Control-Process Analytical Technology for Continuous Biologics Antibody Manufacturing. Processes 2021, 9, 172. https://doi.org/10.3390/ pr9010172

Received: 30 November 2020

Accepted: 14 January 2021

Published: 18 January 2021

Publisher's Note: MDPI stays neutral with regard to jurisdictional claims in published maps and institutional affiliations.

Copyright: (c) 2021 by the authors. Licensee MDPI, Basel, Switzerland. This article is an open access article distributed under the terms and conditions of the Creative Commons Attribution (CC BY) license (https:// creativecommons.org/licenses/by/ $4.0 /)$.
Institute for Separation and Process Technology, Clausthal University of Technology, Leibnizstr. 15, 38678 Clausthal-Zellerfeld, Germany; helgers@itv.tu-clausthal.de (H.H.); schmidt@itv.tu-clausthal.de (A.S.); lohmann@itv.tu-clausthal.de (L.J.L.); vetter@itv.tu-clausthal.de (F.L.V.); juckers@itv.tu-clausthal.de (A.J.); jensch@itv.tu-clausthal.de (C.J.); mouellef@itv.tu-clausthal.de (M.M.); zobel-roos@itv.tu-clausthal.de (S.Z.-R.)

* Correspondence: strube@itv.tu-clausthal.de; Tel.: +49-5323-72-2355
Abstract: Continuous manufacturing opens up new operation windows with improved product quality in contrast to documented lot deviations in batch or fed-batch operations. A more sophisticated process control strategy is needed to adjust operation parameters and keep product quality constant during long-term operations. In the present study, the applicability of a combination of spectroscopic methods was evaluated to enable Advanced Process Control (APC) in continuous manufacturing by Process Analytical Technology (PAT). In upstream processing (USP) and aqueous two-phase extraction (ATPE), Raman-, Fourier-transformed infrared (FTIR), fluorescence- and ultraviolet/visible(UV/Vis) spectroscopy have been successfully applied for titer and purity prediction. Raman spectroscopy was the most versatile and robust method in USP, ATPE, and precipitation and is therefore recommended as primary PAT. In later process stages, the combination of UV/Vis and fluorescence spectroscopy was able to overcome difficulties in titer and purity prediction induced by overlapping side component spectra. Based on the developed spectroscopic predictions, dynamic control of unit operations was demonstrated in sophisticated simulation studies. A PAT development workflow for holistic process development was proposed.

Keywords: quality by design (QbD); process analytical technology (PAT); digital twin; chemometrics; multivariate data analysis; continuous manufacturing; real time release testing (RTRT); Chinese hamster ovary $(\mathrm{CHO})$; monoclonal antibody (mAb); raman spectroscopy; attenuated total reflection fourier-transformed infrared spectroscopy (ATR-FTIR); fluorescence; diode array detector

\section{Introduction}

Innovation in biologics manufacturing urges toward continuous operations to cope with new entities in smaller volumes, such as antibody fragments, virus-like particles (VLPs), exosomes, and mRNA (messenger ribonucleic acid) [1,2]. However, to demonstrate innovation, at first, monoclonal antibody manufacturing in Chinese Hamster Ovary (CHO) cells is the standard platform process [3]. Nonetheless, monoclonal antibody manufacturing in Chinese Hamster Ovary $(\mathrm{CHO})$ cells is still the most widely used process for demonstrating new innovations, as it is well established in industry and academia. In early process development, several important decisions have to be made, e.g., use of disposable or stainless-steel equipment, and any decision has to be filed upon the processing operation, which includes the control strategy within the regulatory demanded quality by-design $(\mathrm{QbD})$ concept. In contrast to documented lot deviations in batch or fed-batch operation, continuous manufacturing opens up new operation windows with improved constant product quality in combination with advanced process control (APC) [4]. However, to achieve APC, more sophisticated sensors are key technologies in a robust control strategy, 
since they can provide additional information on the process state. Moreover, night and weekend shift operation teams have to be supported by reliable process controls. Fully autonomous processing is in high demand.

The unit operations for a fully continuous process have been proposed and their feasibility is well documented [5,6]. Recent improvements focus on digital twins [3] and process control $[7,8]$. Implementation of an advanced control strategy requires sensors, in-line or at-line analytics, which have to be chosen in early process development, e.g., for the chromatography units, which are the key-technology for product purity [7-9].

First, in-line studies started naturally with the first unit operation, cultivation, either operated as a fed-batch or perfusion, with a broad application portfolio [1,10-13]. Nevertheless, the whole downstream has to follow.

Due to its equipment complexity, continuous chromatography has a long tradition in advanced process control concepts [14-16] for autonomous operation. Break-through operations in capturing like periodic counter-current chromatography (PCC) and multicolumn solvent gradient purification (MSCGP) processing $[17,18]$ could easily be controlled by inline UV detection $[19,20]$, since the switch criteria are defined by target component breakthrough. More complex offline analytics and model-based calculations are therefore not necessary, but possible, of course [6]. In addition to UV sum signal detection, diodearray detector (DAD) concepts of peak deconvolution have successfully been applied to the separation of monoclonal antibodies (mAbs) [8,21]. This approach could specify side components at least in main groups to fine-tune the switch criteria if intended.

In general, PAT is not limited to in-line analytics, but is a consistent technology approach that is integrated into the $\mathrm{QbD}$ philosophy demanded by regulatory authorities. It includes process control in order to gain real time release testing (RTRT) as a benefit in quality assurance (QA) efforts reduction as improved product quality. RTRT has to correlate to critical product quality attributes, such as bio-efficacy by titer, purity, and bioactivity. State of the art QA are offline analytical methods, such as Protein A and size exclusion chromatography (SEC), enzyme-linked immunosorbent assay (ELISA), infrared spectroscopy, as well as glycosylation analytics via HPLC or HPLC-mass spectrometry [22-24]. The feasibility of RTRT by online PAT tools needs to be proven.

For process development, a sophisticated PAT concept has to be developed parallel to upstream processing (USP) and downstream processing (DSP) modeling, later on supporting model validation [3,25-29], piloting, and production, as shown in Figure 1. Parallel to model validation, piloting, and production the developed PAT method and the partial least squares regression (PLSR) system have to be further refined. In addition to appropriate PAT, digital twins for the whole process are a central key-technology for achieving RTRT. It has been proven that, for all unit operations, such distinct validated process models are available as digital twins $[1,5,8,26,27,29-32]$.

\subsection{State of the Art in Spectroscopic PAT}

In Table 1 an overview of different spectroscopic methods is given. Raman spectroscopy is a promising candidate to enable measurements in impure samples, e.g., by using an in-situ probe in upstream processing [12,33,34]. In Raman spectroscopy, due to inelastic impacts of photons with the analytes, the photons are scattered [34]. The scattering pattern is molecule-specific and, therefore, the identification and quantification of different components is possible. Raman spectroscopy was successfully employed in USP for the quantification of substrates (e.g., glucose), metabolites (e.g., lactate), and mAb [12,35]. In contrast to alternative processes, such as aqueous two-phase extraction (ATPE) and precipitation, in which barely any spectroscopic methods have been published for explicit use as PAT, various concepts have already been demonstrated for established processes such as chromatography, ultrafiltration/diafiltration (UF/DF) and lyophilization [36,37]. 


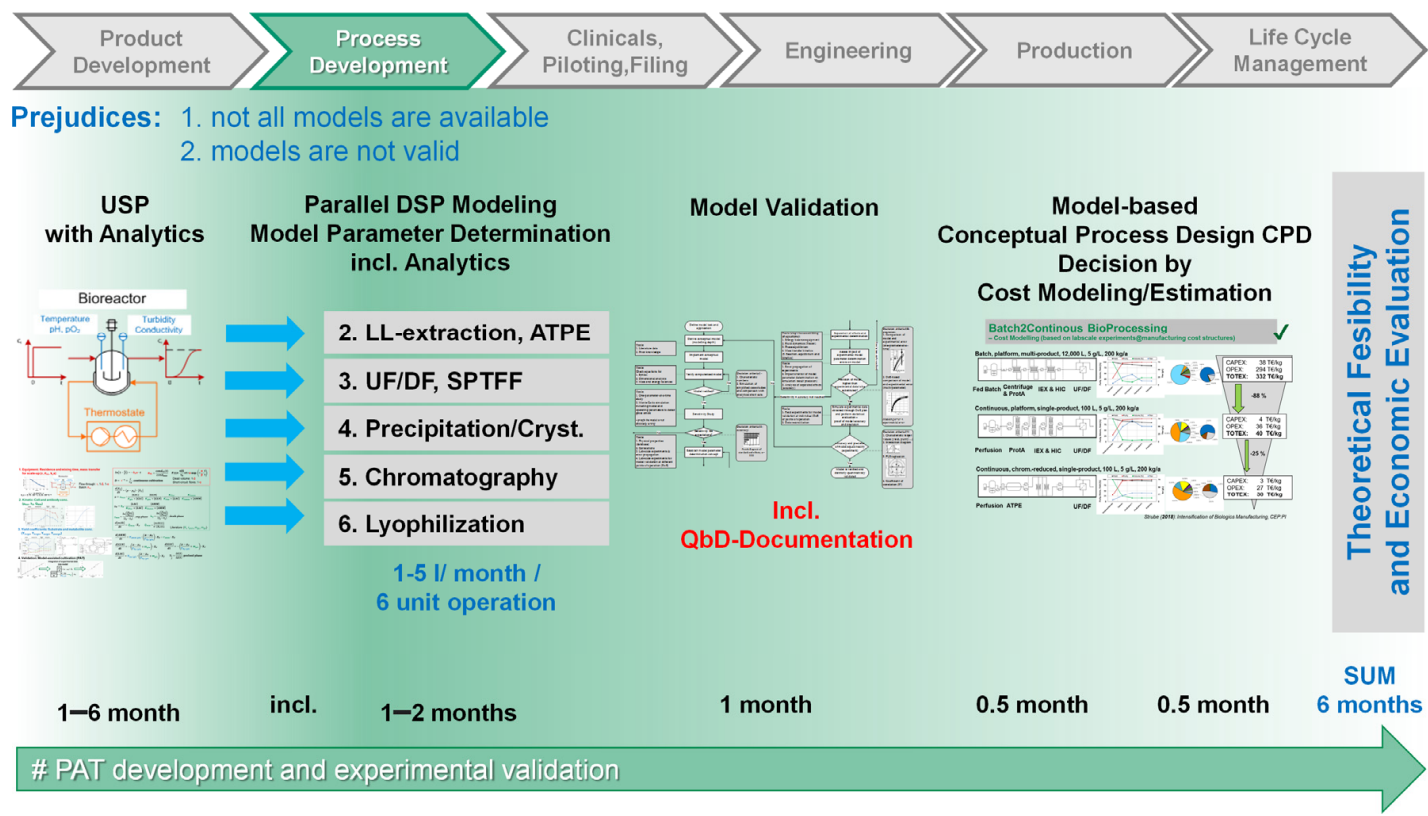

Figure 1. Process development strategy. The development of a new biologics manufacturing process starts with screening for optimal cultivation conditions in upstream processing (USP). Samples from optimization experiments are used for establishing Process Analytical Technology (PAT), partial least squares regression (PLSR), and physicochemical processmodels in USP. In parallel first downstream processing (DSP)-optimization experiments are performed. Models have then to be validated according to published validation workflows that ensure accuracy and precision of modeling results.

Fourier-transformed-infrared-spectroscopy (FTIR) measures the absorption of photons, typically between $4000-400 \mathrm{~cm}^{-1}$. Biomolecules contain many amine, carbonyl or hydroxy groups, which absorb photons in this low-energy range by inducing stretching, scissoring and bending of molecule bonds [38]. In contrast to Raman spectroscopy, in IR spectroscopy the absorption from water is considerably strong and may interfere with concentration measurement of biomolecules in aqueous solution [39]. Nonetheless, IR measurement was successfully applied in USP for the prediction of $\mathrm{mAb}$ concentration [40]. While applicable in early process stages, FTIR is also a viable technique for quality evaluation [22,41]. FTIR was successfully applied in protein detection in ATPE [42], monitoring of $\mathrm{mAb}$ purification in chromatography [43] and inline concentration measurement in ultrafiltration [37]. Raman and NIR can be used as PAT technology in lyophilization processes [44-50]. Raman spectra can indicate different critical product and process characteristics, e.g., water to ice conversion, product crystallization, annealing steps, solid-state characteristics of intermediate and end products and kinetics of polymorphic transitions [49,50]. NIR can also indicate critical product and process characteristics [50] such as the secondary structure of lyophilized proteins and the residual moisture [49]. NIR and Raman provide the ability to determine the endpoint of primary drying. Water and ice produce weak Raman signals but have high absorption in NIR spectra. Both measurements determine the endpoint by detecting a loss of water signal, but NIR is the more sensitive technique and is additionally capable of detecting the endpoint of secondary drying [44,50]. DAD measures the absorption of photons in UV/VIS, typically in the range between 190-520 nm. In contrast to FTIR, in this high-energy range, delocalized $\pi$-electron systems are the main absorbers [51]. DAD is widely used in chromatography but potential applications in earlier process stages exist [7]. Fluorescence measures the time-delayed emission of photons after excitation by a specific wavelength. The emission range is lower than the excitation wavelength, since the photons lose energy after absorption due to non-radiative transitions [52]. Fluorescence was previously employed in monitoring upstream processes [10]. Integrity of IgG can also be monitored by fluorescence, e.g., in combination with circular dichroism measurement [53]. The limits of detection values given in Table 1 are for orientation purposes only, since they vary with 
different measurement/integration times and product. However, the presence of too many side components can reduce the detection accuracy [54]. Detection accuracy enhancements through combination of multiple spectroscopic techniques have been proposed [55].

The above presented PAT approaches in combination with a digital twin can be used to achieve advanced process control and in-line process optimization [30,56-60]. PAT can compensate model inaccuracies by providing additional measurement data and the digital twin is used for inline process optimization and online process monitoring.

Table 1. Overview of measurement parameters for Raman, Fourier-transformed infrared (FTIR) diode-array detector (DAD) and fluorescence from literature and manufacturers.

\begin{tabular}{ccccccc}
\hline Detector & $\begin{array}{c}\text { Measurement } \\
\text { Range }\end{array}$ & In Situ Probes & Flow Cell & $\begin{array}{c}\text { Lower Limit of } \\
\text { Detection }\end{array}$ & $\begin{array}{c}\text { Acquisition } \\
\text { Time }\end{array}$ & $\begin{array}{c}\text { Averaged } \\
\text { Scans }\end{array}$ \\
\hline Raman & $4000-400 \mathrm{~cm}^{-1}$ & Available & $380 \mu \mathrm{L}$ & $>50 \mathrm{mg} / \mathrm{L}[61]$ & $10 \mathrm{~s}[12]$ & $75[12]$ \\
FTIR & $4000-400 \mathrm{~cm}^{-1}$ & Available & - & $>700 \mathrm{mg} / \mathrm{L}[43]$ & $4 \mathrm{~s}[62]$ & $16-64[40,62]$ \\
DAD & $190-520 \mathrm{~nm}$ & Unavailable & $8 \mu \mathrm{L}$ & $>10 \mathrm{mg} / \mathrm{L}[63]$ & $0.1 \mathrm{~s}[7]$ & - \\
Fluorescence & $280-900 \mathrm{~nm}$ & Unavailable & $16 \mu \mathrm{L}$ & $>40 \mathrm{fg} / \mathrm{L}[64]$ & $3 \mathrm{~s}[53]$ & $10[65]$ \\
\hline
\end{tabular}

\subsection{QbD-Based PAT Control Strategy}

In general, PAT is not limited to in-line analytics, but is a consistent technology approach, which is integrated into the $\mathrm{QbD}$ philosophy, demanded by regulatory authorities and is becoming the standard in biopharmaceutical process development. In the $\mathrm{QbD}$ approach, a design space of operating parameters is defined, to ensure specified quality attributes (QAs). This leads to multi-parameter optimizations and a significant experimental effort. The modern approach for process development and quality assessment is shown in Figure 2.

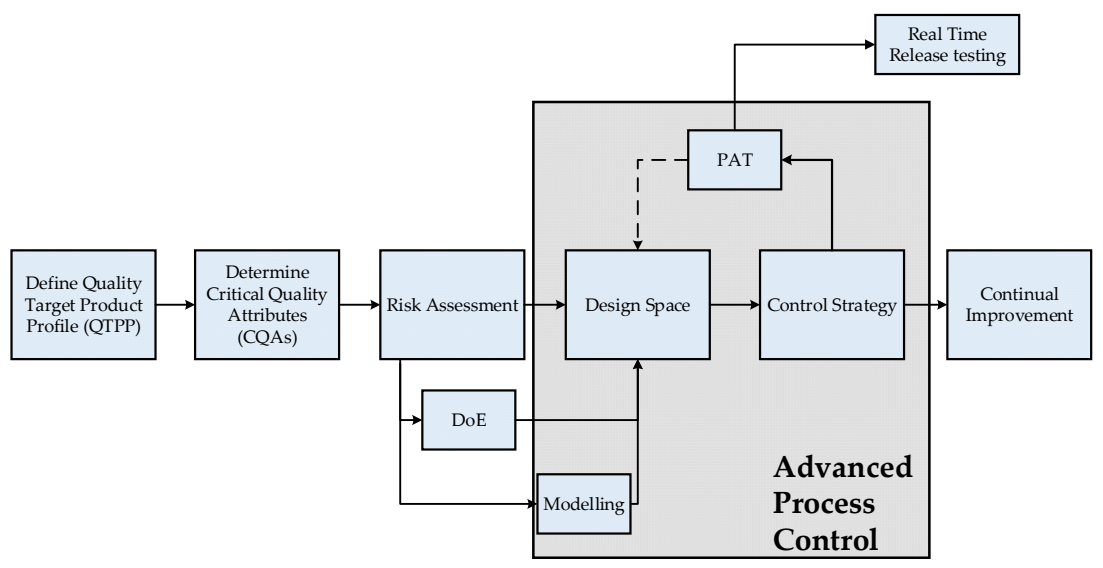

Figure 2. Quality by-design (QbD)-based process development workflow. Firstly, the quality target product profile is defined and critical quality attributes are determined. Next, a risk assessment serves as starting point for design of experiments (DoE) and/or modeling, which outcome then defines the design space. During process operation, PAT provides real time measurement of critical process parameters that are then compared to the limits of the design space. To ensure that the process continuously operates within the boundaries of the design space, process models can utilize real time PAT measurements to calculate necessary process adjustments.

One of the most utilized APC concepts are model predictive controllers (MPC) [66]. These MPCs manipulate input variables to match the desired set points, while maintaining process critical constraints. This is performed by utilizing optimization routines on process models, which predict the future process behavior for the next time frame [67,68]. Common drawbacks of these models are that the model results tend to drift away from the real plant data over time, because of aging, fouling, or blocking phenomena, or summation 
of prediction errors in cyclic processes [69]. This is usually fixed by updating the internal model states, e.g., concentrations, with real plant data [70]. This real time plant data have to be determined via potentially time intensive and invasive offline analytics, if no PAT tools are implemented, resulting in a gap between current process data and analytics [71,72]. This gap not only risks a mismatch between current process state and model, but also a general mismatch between current process and process analytics that prevents data-driven process decisions, especially in continuous processes.

Starting with this overview, we will demonstrate that PAT is capable of filling the gap. The proposed control strategy is demonstrated exemplarily in simulation studies. Previous studies have focused on the implementation and distinct and quantitative validation of physico-chemical process models to describe the unit operations shown in Figure 3, and enable advanced process control. The successful operation of the proposed continuous process has been previously shown [1]. The missing link to achieve APC is a holistic PAT strategy [5]. The proposed control strategy is demonstrated exemplarily in simulation studies.

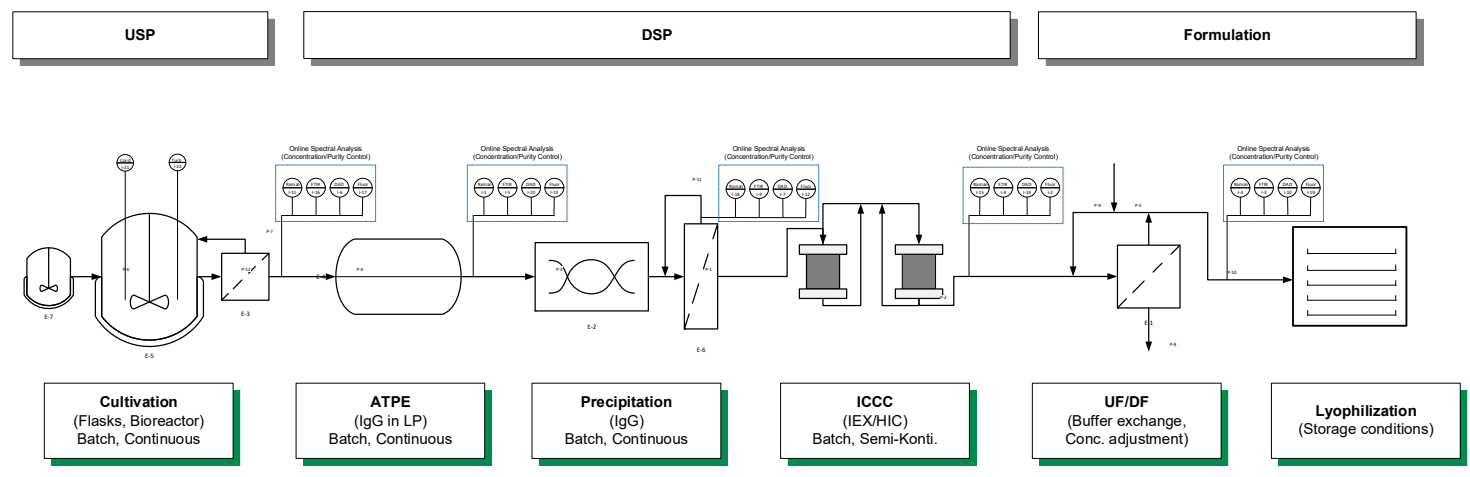

Figure 3. Proposed process flow sheet including spectroscopic PAT sensors. A PAT measurement array is placed at the outlet of each unit operation to provide real time data on monoclonal antibody (mAb), high molecular weight (HMW) impurities, and light molecular weight (LMW) impurity concentrations that are then used as input for the process model, which calculates the necessary process adjustments to ensure operation within a pre-defined design space.

The presented study is an introducing overview to a series of publications showing the holistic methodology for the development of a spectroscopy-based PAT for APC. The focus here is therefore on the overall development with the important steps of spectra acquisition, spectra processing, and evaluation of the achievable prediction accuracy, especially in comparison to established offline analytics, and finally the conceptualization as well as the proof-of-concept of a PAT-based APC through simulation studies.

The aim is therefore not to present only the APC as a final result, but to present the necessary steps and decision criteria.

This demonstration of feasibility, which is necessary as a missing link, has been provided for the first time in the completeness of the development, the unit operations, and the possible spectroscopy technology presented here.

\section{Materials and Methods}

Chinese hamster ovary cells (CHO DG44) producing an industrially relevant monoclonal antibody $(\mathrm{mAb})$ from the IgG1 subclass were cultivated as described elsewhere [5].

Phase forming components used are harvested cell culture fluid (HCCF), polyethylene glycol with an average molecular weight of $400 \mathrm{Da}$ (Merck KGaA, Darmstadt, Germany) and phosphate. The salt buffer solution consists of $262.09 \mathrm{~g}$ disodium phosphate dihydrate (Merck, Darmstadt, Germany), 198.39 g potassium dihydrogen phosphate (Merck, Darmstadt, Germany) and $539.52 \mathrm{~g}$ deionized water.

Precipitation procedure was conducted similar to batch operation described by Lohmann et al. [29]. After dissolution, the $\mathrm{pH}$ was adjusted to 3.7 and held for $60 \mathrm{~min}$ for virus inactivation [73]. Precipitation and dissolution were investigated separately with 
respect to the different spectroscopic analysis methods (Raman, FTIR, DAD, fluorescence) and PAT sensors ( $\mathrm{pH}$, turbidity, conductivity). In precipitation and dissolution, the same online detection train is used as described in ATPE with the exception of the fluorescence detector, which is not used in precipitation due to precipitates, which are harming for the flow cells of the fluorescence detector.

Figure 4 shows the experimental set-up, on the example of ATPE. The analytical procedure (green) and the offline analytics (grey) were the same for all unit operations.

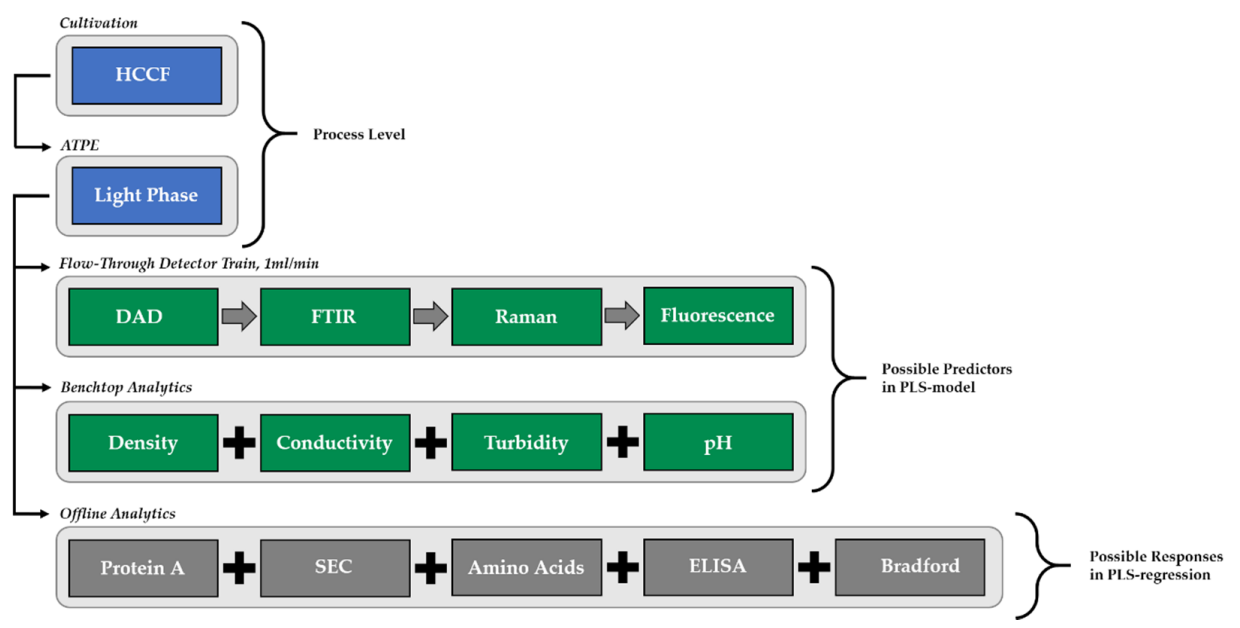

Figure 4. Experimental set-up (blue) and analytical procedure (green). Harvested cell culture fluid (HCCF) is from start $(\mathrm{t} 0)$ to end of cultivation ( $\mathrm{t} 10)$ processed by aqueous two-phase extraction (ATPE). The antibody containing light phase is pumped in the detector array for spectral analysis and investigated in PLSR as predictors. Offline analytics (grey) are investigated as responses.

The dissolved antibody obtained after precipitation was used in the chromatography studies. Purification was accomplished using a YMC S75 strong cation-exchange column (YMC-BioPro S75, $26 \times 7.0$ mm ID, YMC Co., Ltd., Kyoto, Japan). The starting solution was loaded on the column for 5 column volumes (CV). For flow-through-FTIR and Raman, $30 \mathrm{CV}$ were loaded on the column.

Samples for spectral analysis of UF/DF were prepared by mixing appropriate amounts of a $165 \mathrm{~g} / \mathrm{L}$ polyclonal antibody solution (Gammanorm, Octapharma AG, Lachen, Switzerland) with the expected buffer composition of the respective filtration stage. Table 2 shows the prepared solutions with the respective $\mathrm{mAb}, \mathrm{NaH}_{2} \mathrm{PO}_{4}$, and $\mathrm{NaCl}$ concentrations.

Table 2. Prepared $\mathrm{mAb}$ samples with expected salt concentrations for theoretical ultrafiltration/diafiltration (UF/DF) stages.

\begin{tabular}{ccccccc}
\hline Substance & Stage $\mathbf{1}$ & Stage $\mathbf{2}$ & Stage $\mathbf{3}$ & Stage $\mathbf{4}$ & Stage 5 & Stage 6 \\
\hline $\mathrm{mAb}(\mathrm{g} / \mathrm{L})$ & 0 & 2 & 9 & 13 & 18 & 20 \\
$\mathrm{NaH}_{2} \mathrm{PO}(\mathrm{mM})$ & 50 & 50 & 34 & 23 & 12 & 6 \\
$\mathrm{NaCl}(\mathrm{mM})$ & 1000 & 1000 & 625 & 375 & 125 & 0.1 \\
\hline
\end{tabular}

PLSR models were built for three different analytes: target component (TC), concentration of high molecular weight (HMW) impurities, and light weight molecular weight (LMW) impurities.

\subsection{Analytics}

\subsubsection{Online}

Data acquisition in Raman spectroscopy (785 nm laser, $1.5 \mathrm{~mW}$, Ocean Insight, Ostfildern, Germany, with an InPhotonics Raman probe) was set to automatically average a total of three spectra, each of which had $1 \mathrm{~s}$ of integration time. For each sample, at least 
three spectra were measured and averaged prior to PLSR. Data acquisition in FTIR spectroscopy (Alpha II, Bruker, Billerica, MA, USA) was set to automatically average a total of 24 spectra, each of which had $1 \mathrm{~s}$ of integration time. Data acquisition in UV-Vis spectroscopy, using a DAD (Smartline DAD 2600, Knauer Wissenschaftliche Geräte GmbH, Berlin, Germany), was set to continuously record at a sampling rate of $0.2 \mathrm{~s}^{-1}$, each of which had $32 \mathrm{~ms}$ of integration time. Data acquisition in fluorescence spectroscopy (Jasco FP-2020 Fluorescence detector) was set with a gain factor of 1 . For each sample, at least three spectra were measured and averaged prior to PLSR. Data acquisition in fluorescence spectroscopy was measured using a gain of 10 .

\subsubsection{Offline}

Samples for off-and at-line analyses were drawn at least once per day. The viable cell concentration was determined using a Cedex XS (Roche Innovatis AG, Bielefeld, Germany), with the trypan blue exclusion method using a diluted trypan blue stock solution $(0.4 \%$, Sigma-Aldrich, St. Louis, MO, USA). Cells were separated by centrifugation at $4000 \mathrm{rcf}$ and the cell free supernatant was either used directly for analysis or stored at $-20^{\circ} \mathrm{C}$ before it was analyzed. Glucose and lactate concentrations were determined by enzymaticamperometric measurement using a LaboTRACE compact (TRACE Analytics GmbH, Braunschweig, Germany). The monoclonal antibody concentration was quantified by Protein A chromatography (PA ID Sensor Cartridge, Applied Biosystems, Bedford, MA, USA). Dulbecco's PBS buffer (Sigma-Aldrich, St. Louis, MO, USA) was used as loading buffer at $\mathrm{pH} 7.4$ and as elution buffer at $\mathrm{pH}$ 2.6. Size exclusion chromatography (SEC) was done with a YarraTM $3 \mu \mathrm{m}$ SEC 3000 column (Phenomenex Ltd., Aschaffenburg, Germany) utilizing $0.1 \mathrm{M} \mathrm{Na}_{2} \mathrm{SO}_{4}, 0.1 \mathrm{M} \mathrm{Na}_{2} \mathrm{HPO}_{4}$, and $0.1 \mathrm{M} \mathrm{NaH}_{2} \mathrm{PO}_{4}$ (Merck KGaA, Darmstadt, Germany) as a buffer system. The absorbance for both methods was monitored at $280 \mathrm{~nm}$. Retention of biological activity is shown via a target specific ELISA (ELISA Assay, Eagle Bioscience, NH, USA). Two-dimensional (2D)-SDS PAGE was conducted for visualization of side components and their elimination progress during downstream processing. For isoelectric focusing, IPG strips (ReadyStripTM IPG Strips, linear, pH 3-10, BIO-RAD, Hercules, CA, USA) was well as a power supply from Hoefer (Hoefer Inc., Holliston, MA, USA) were used. Subsequent, SDS-Page was carried out utilizing gels (Criterion TGX Precast Gel, 4-15\% Bis-Tris, Bio-Rad), buffers, and electrophoresis chamber from Bio-Rad. Coomassie Brilliant Blue G-250 (VWR International, Radnor, PA, USA) was used as dye. Fluorescence spectra were measured at-line using a Jasco FP-2020 fluorescence detector. Inline measurement using this detector was not possible, since a spectra measurement takes about $30 \mathrm{~s}$. Gain was set to 1 or 10, depending on the concentration. Using a gain of 10 , a concentration of up to $0.3 \mathrm{~g} / \mathrm{L}$ was practically measurable. Attenuation was set to 256 . Fluorescence measurements were done using the $16 \mu \mathrm{L}$ flow-cell provided with the detector. For each unit operation, emission spectra were measured using an excitation wavelength of $280 \mathrm{~nm}$. Spectra were obtained from 280 to $900 \mathrm{~nm}$. Bradford assays were performed using a Bradford assay kit (Thermo Fisher Scientific, Dreieich, Germany), according to the manufacturer's instructions. Tests were performed in UV-transparent cuvettes and the absorbance at $280 \mathrm{~nm}$ was measured using a UV/Vis SmartSpec Plus Spectrophotometer (Bio-Rad Laboratories GmbH, Feldkirchen, Germany). DNA concentration determination was performed using the Quant-iT ${ }^{\mathrm{TM}}$ PicoGreen ${ }^{\mathrm{TM}}$ dsDNA Assay Kit according to the manufacturer's instructions (Thermo Fisher Scientific GmbH). Samples were measured in flow-through using the Jasco FP-2020 Fluorescence detector with a gain factor of 10 .

\subsection{Analysis of Spectral Data}

Spectra were processed and analyzed using Unscrambler ${ }^{\circledR}$ X (Camo Analytics AS, Oslo, Norway). The raw spectra were analyzed by means of descriptive statistics in order to decide on a suited preprocessing strategy. A main tool was the plot of the spectra against the mean spectrum, also known as scatter effects plot, which shows the nature of distorting effects present in the spectra. Depending on the kind of effect, the 
preprocessing was causally adapted to extract a maximal information content. Figure 5 shows exemplary scatter effects plots of (a) additive effects, (b) scatter effects, (c) additive and scatter effects, and (d) complex effects. Additive effects were eliminated by applying a baseline correction and/or a derivation. If not stated otherwise, first derivatives were taken. Multiplicative scatter effects were removed by applying the method of standard normal variate or multiplicative scatter correction (MSC). Combinations of additive and multiplicative effects were removed by a combination of baseline correction and scatter correction. Effects of the complex type were not observed, though they could be removed by using extended multiplicative scatter correction (EMSC). Preprocessed spectra were then used to correlate the changes in component concentration to the changes in spectral intensity at specific spectral regions by PLSR. A detailed description of the fundamentals of PLSR been published by Esbensen et al. [74]. A maximum of four principal components was allowed in the regression in order to not overfit the data. The quality of the model was evaluated by means of spectral line loadings plot, score plot, and the plot of explained variance against number of principal components.

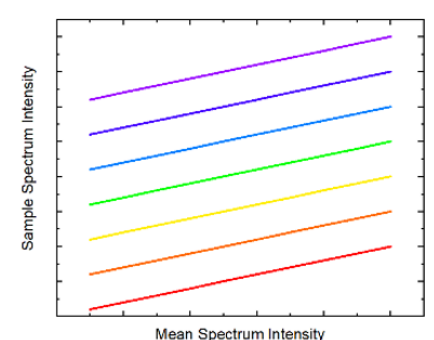

(a)

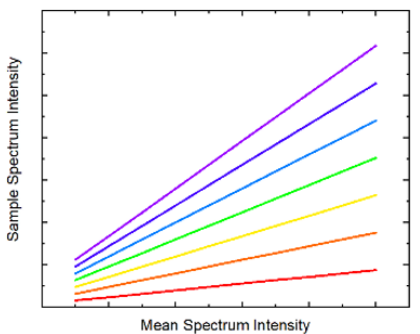

(b)

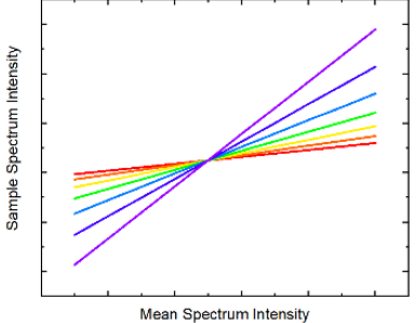

(c)

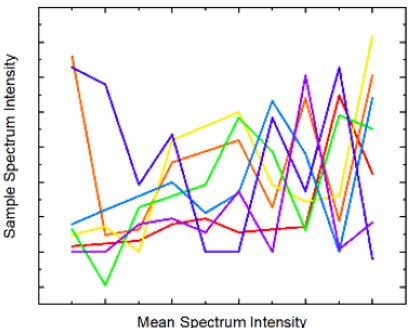

(d)

Figure 5. Exemplary scatter effects plots of (a) additive effects, (b) scatter effects, (c) additive and scatter effects, and (d) complex effects. Colors indicate spectra from different samples.

\subsection{Process Simulation Studies to Propse an Advanced Process Control Strategy}

Since undesired changes of flow rates, titer or deviations of critical quality attribute occur during downstream processing and since the PAT initiative has created a possible tool to detect these changes in real time, the main question is how any unit operation within the whole process can react to these deviations-and how to control them best. Based on the available digital twins for the whole process, this study deals with finding out which fluctuations can be compensated best at which point in the process. Further, it is discussed whether units are able to provide constant output parameters in $\mathrm{mAb}$ concentration (target component, TC), purity and flow rate for the following unit. Therefore, three theoretical scenarios with varying input variables were examined: concentration deviations, volume flow fluctuations and purity changes. Mean parameters are mAb titer at $2 \mathrm{~g} / \mathrm{L}$ and a perfusion flow rate of $1 \mathrm{~mL} / \mathrm{min}$. Purity differs in each unit operation and is adjusted separately. The simulation was conducted with process models for each unit (USP, ATPE, Precipitation, Chromatography) [1,3,25-29] executed in Aspen Custom Modeler ${ }^{\mathrm{TM}}$ (ACM).

\section{Results}

\subsection{Raman Spectroscopy}

All spectra were preprocessed before a PLSR was performed. Raman- and FTIR spectra were trimmed to the relevant (fingerprint) region for $\mathrm{mAbs}$ and relevant side components, which was $1800-400 \mathrm{~cm}^{-1}$, and $1800-900 \mathrm{~cm}^{-1}$, respectively. DAD and fluorescence spectra were used in their full width, i.e., from 190-520 and 280-900 nm, respectively. Spectra were analyzed by means of descriptive statistics as described above. In USP, the scatter effects plots revealed primarily additive effects, independent of the detector. According to the proposed workflow, additive effects were removed by calculating first order derivatives using the Savitzky-Golay derivative method with a polynomial order of two and seven 
smoothing points per side (i.e., 15 points total). Total Raman-spectral intensity, which is primarily caused by fluorescence, decreases during the first three days, after which it constantly increases. This can be attributed to a decreasing concentration of substrates during the batch phase, while the overall protein concentration only slowly starts to increase. After $72 \mathrm{~h}$ the daily feed is started, and the protein concentration starts to rise proportionally (i.e., exponentially) to the total cell concentration, thereby increasing the overall fluorescence (cf. Figure 6). Preprocessed spectra show increases in the amide I, II and III band intensities that are in line with the protein concentration increase. Moreover, pronounced intensities at $\sim 500,850$ and $950 \mathrm{~cm}^{-1}$ were observed, which correspond to C-C-O bending $\left(540 \mathrm{~cm}^{-1}\right)$, C-COO stretching $\left(855 \mathrm{~cm}^{-1}\right)$ and $\mathrm{CH}_{3}$ rocking $\left(930 \mathrm{~cm}^{-1}\right)$, respectively [12].

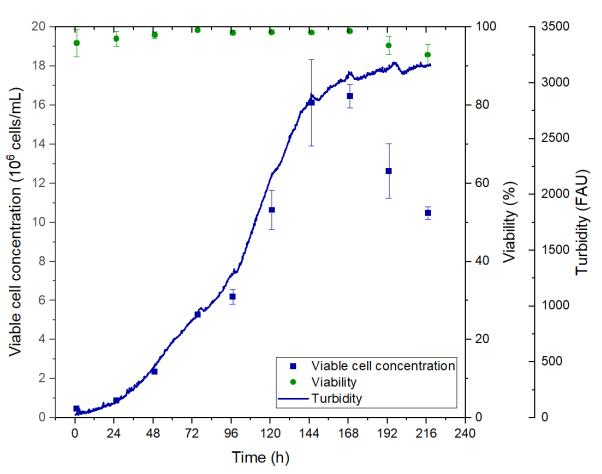

(a)

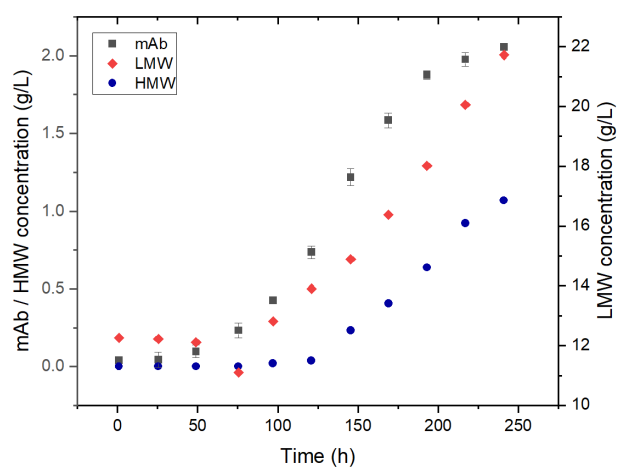

(b)

Figure 6. (a) Viable cell concentration, viability and turbidity over process time. (b) mAb concentration and HMW/LMW pseudo-concentrations over process time.

PLSR was used to correlate changes in Raman spectral intensity with regards to the target component mAb concentration and typically defined HMW (high molecular weight host cell protein fraction) and LMW ((low molecular weight host cell protein fraction) pseudo-concentrations as these are the relevant quantities in the following DSP. Hence, these concentrations should be constantly measured and forwarded to the following unit operation in a continuous process that is controlled by APC. The regression of the predicted vs. the actual concentration yields a good correlation of $\mathrm{R}^{2}=0.999,0.994$, and 0.998 for $\mathrm{mAb}, \mathrm{HMW}$ and LMW concentrations, respectively, using one principal component. The highest deviations occur at low concentrations, e.g., for mAb at concentrations smaller than $0.3 \mathrm{~g} / \mathrm{L}$, while higher concentrations can be predicted more accurately. RMSE values were $0.028,0.029$ and $0.139 \mathrm{~g} / \mathrm{L}$, for $\mathrm{mAb}, \mathrm{HMW}$ and LMW concentrations, respectively. This corresponds to a deviation of $1.4 \%, 2.7 \%$, and $0.6 \%$ in regard to the final for mAb, HMW and LMW concentrations, respectively.

Raw Raman spectra in ATPE show strong additive effects, which increase with cultivation time. Especially in the fingerprint region between $1800-400 \mathrm{~cm}^{-1}$, there is a strong baseline increase. This can be mainly attributed to the concentration increase of proteins. As can be seen after transformation by deviation and normalization, larger differences in the spectra can be identified at amide I $\left(1650 \mathrm{~cm}^{-1}\right)$, amide II $\left(1550 \mathrm{~cm}^{-1}\right)$ and amide III $\left(1300 \mathrm{~cm}^{-1}\right)$ bands. Overall, PLSR shows that in a range of $0-2 \mathrm{~g} / \mathrm{L} \mathrm{mAb}$ a titer prediction accuracy of $\mathrm{R}^{2}=0.97$ is achieved, when compared to offline protein A determination. The RMSE of prediction was $0.075 \mathrm{~g} / \mathrm{L}$, which is $4 \%$ of the final $\mathrm{mAb}$ concentration. The error is similar to offline Protein A chromatography. HMW prediction results in a smaller regression coefficient of $\mathrm{R}^{2}=0.87$, when compared to SEC based offline determination, with a RMSE of $0.013 \mathrm{~g} / \mathrm{L}$, or $8 \%$ of the final HMW pseudo-concentration. As ATPE produces light phase that throughout the process is characterized by high HMW purity (92\%), the HMW concentration is also small compared to the $\mathrm{mAb}$. It is therefore to be expected that the combination of low concentration and intrinsic fluorescence makes the identification of HMW related changes in the Raman spectra more difficult. LMW prediction on the other hand results in a regression coefficient of $R^{2}=0.96$ (also regressed against SEC determina- 
tion), with a RMSE of $0.131 \mathrm{~g} / \mathrm{L}$ or $1 \%$ of the final LMW pseudo-concentration. After ATPE the LMW purity in the light phase throughout the process is small (11.6\%), so the LMW concentration is high and although LMW consist of wide range of different species, such as proteins, peptides, vitamins etc., their summarized contribution to the overall Raman spectra is different enough to the mAb contribution to enable a PLSR.

In precipitation, the Raman intensity decreases for a higher PEG content. With increasing PEG content more side components co-precipitate and increase in this manner particle density. A similar trend can be observed in Raman raw data for dissolution. Raman intensity decreases with increasing concentration of redissolved $\mathrm{mAb}$. Further preprocessing was applied after evaluation of scatter effects. Spectra were trimmed to $1800-400 \mathrm{~cm}^{-1}$ and the first derivative was taken using the Savitzky-Golay method with three smoothing points per side.

In the case of Raman, preprocessing was equal for precipitation and dissolution. The LMW pseudo-concentration could be well correlated to changes in spectra with an $\mathrm{R}^{2}$ of 0.97 and RMSE of $0.03 \mathrm{~g} / \mathrm{L}$. In dissolution, the concentration of $\mathrm{mAb}$ could be predicted with an $\mathrm{R}^{2}$ of 0.93 and a RMSE of $0.08 \mathrm{~g} / \mathrm{L}$. These deviations are equivalent to $0.3 \%$ for LMWs in precipitation and $3.9 \%$ for the final $\mathrm{mAb}$ concentration in dissolution. It can be concluded that Raman is a suitable detector for precipitation and dissolution.

In chromatography the first derivative of spectral data are calculated. PLSR validation for Raman spectroscopy in chromatography was not possible, as shown in Figure 7. The reason for this is that the large flow-cell in combination with the low flow rate results in a long retention time of the product causing back-mixing. This is illustrated in Figure 8. Here, the results of a tracer experiment with (red) and without (black) are depicted. The widened peak after the flow cell is a product of back-mixing occurring in the flow cell. The RMSE value for Raman is 4.7 , corresponding to $\sim 80 \%$ of the highest value in the Raman samples.

In UF/DF additive effects were observed in raw Raman-spectra. Accordingly, first derivatives were calculated from the raw spectra and a PLSR was performed, yielding a correlation of $\mathrm{R}^{2}=0.951$, with a RMSE of $1.08 \mathrm{~g} / \mathrm{L}$. At the final concentration of $20 \mathrm{~g} / \mathrm{L}$ the deviation is $5.4 \%$, which is similar to offline analytical methods such as protein A chromatography.

\subsection{Fourier-Transform Infrared Spectroscopy}

FTIR and Raman spectroscopy are often referred to as being complementary techniques. While Raman detects the molecules fingerprint signature of inelastic bonds by scattering photons when excited by a laser, FTIR detects the molecules fingerprint signature of bonds that absorb infrared radiation. Both detection principles however cause the bonds within the molecule to convert some of that energy into vibration, and are therefore summarized as vibrational spectroscopy methods. FTIR spectra were measured against air as background. Hence, all spectra have a common offset from water. Compared to Raman spectroscopy, the absorption of water in IR-measurements is strong, and thus overlays the relevant signals from the target components (i.e., $\mathrm{mAb}$ and side components). FTIR spectra were trimmed to the relevant (fingerprint) region for $\mathrm{mAbs}$ and relevant side components, which was $1800-900 \mathrm{~cm}^{-1}$. In order to remove the offset and make relevant information accessible, the first derivative is calculated. Thereby, the background water signal is removed and two strong peaks can be identified at around $1650 \mathrm{~cm}^{-1}$ corresponding to the amide I band. In contrast to Raman spectroscopy, amide II and amide III bands are less pronounced. PLSR of the mAb, HMW, and LMW concentrations was performed, but yielded generally lower regression coefficients than those in Raman spectroscopy which can partly be attributed to less pronounced amide II and III signals. Moreover, compared to Raman spectroscopy, the overall number and intensity of signals that can clearly distinguished is markedly lower which hampers the prediction. The coefficients of determination for $\mathrm{mAb}$, HMW and LMW are 0.983, 0.986 and 0.989, with RMSEs of 0.102, 0.045, and $0.353 \mathrm{~g} / \mathrm{L}$, respectively. These deviations are equivalent to $5.1 \%, 4.2 \%$, and $1.6 \%$ of the final $\mathrm{mAb}$, HMW, and LMW concentrations, respectively. 


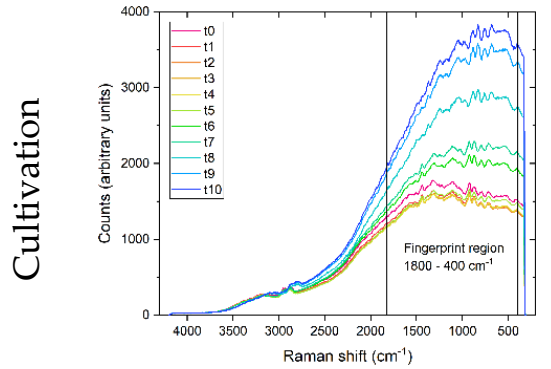

(a)

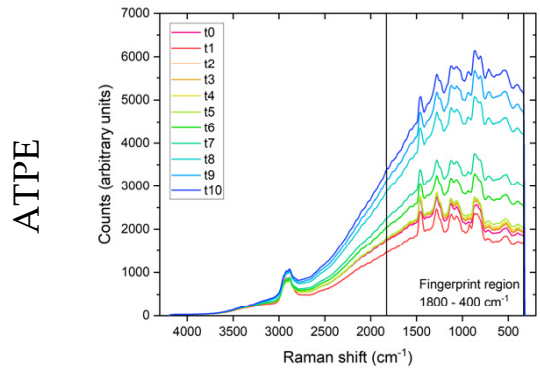

(d)

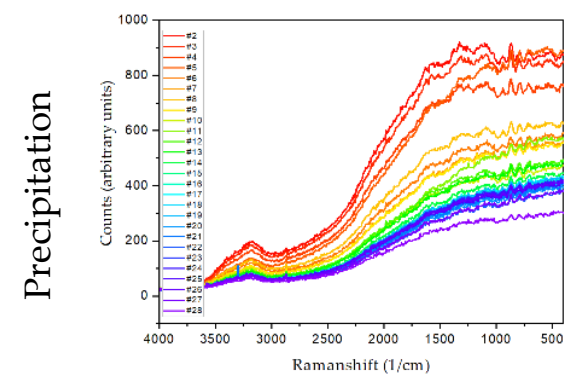

(g)

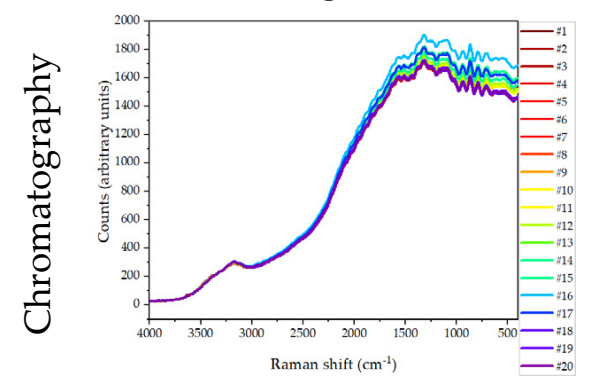

(j)

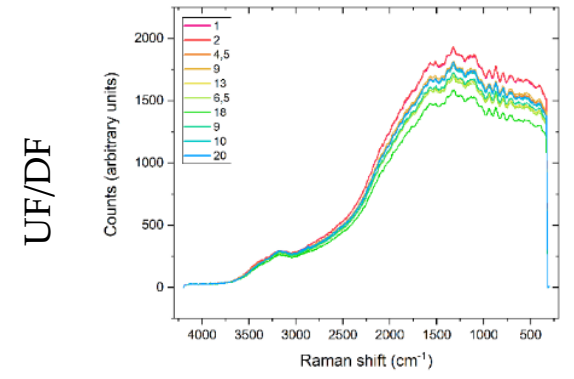

(m)

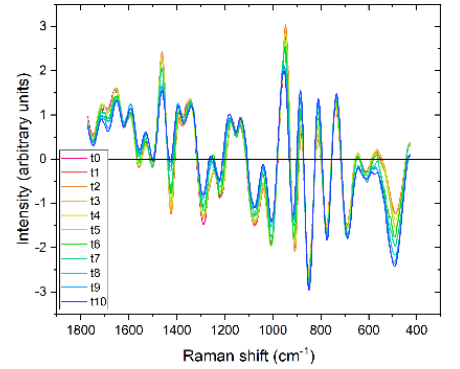

(b)

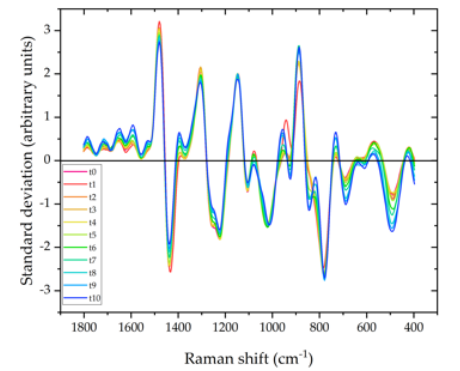

(e)

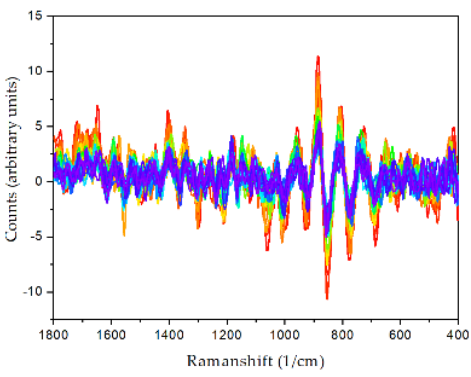

(h)

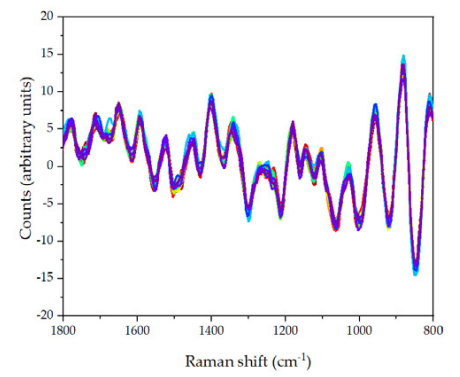

(k)

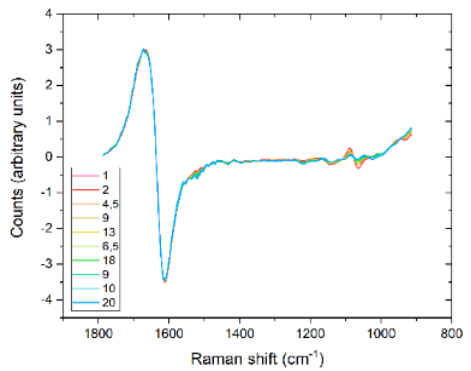

(n)

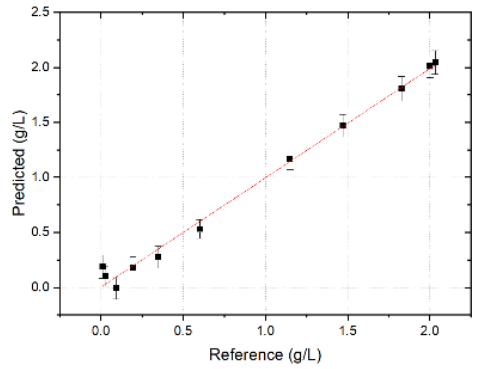

(c)

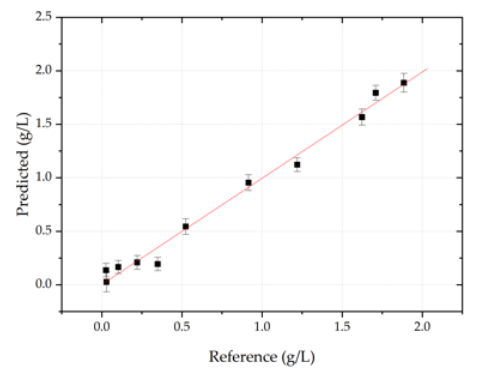

(f)

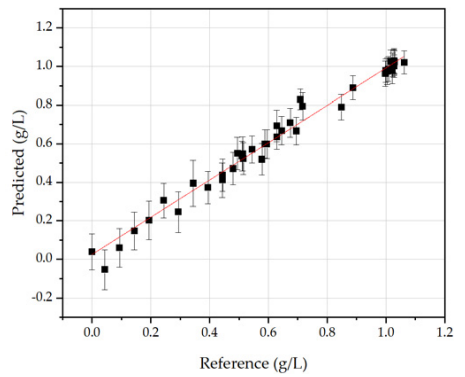

(i)

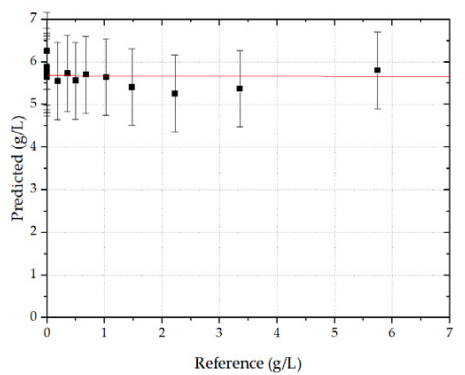

(l)

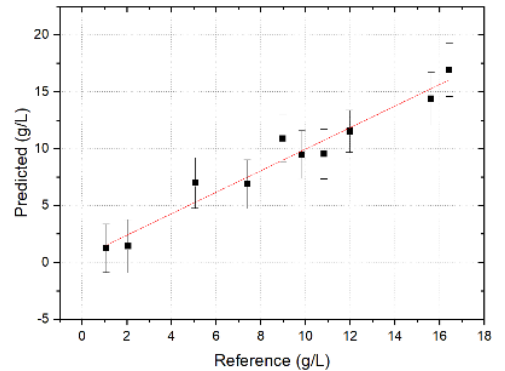

(o)

Figure 7. Raman spectra: raw (a,d,g,j, $\mathbf{m})$, after preprocessing $(\mathbf{b}, \mathbf{e}, \mathbf{h}, \mathbf{k}, \mathbf{n})$ and PLSR results $(\mathbf{c}, \mathbf{f}, \mathbf{i}, \mathbf{l}, \mathbf{o})$. Spectra are color-coded from high concentration (blue) to low concentration (red). 


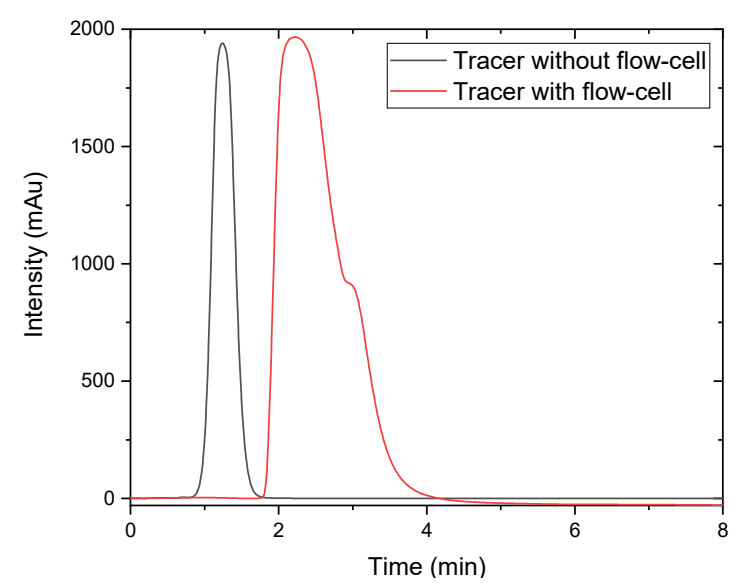

Figure 8. Tracer chromatogram with and without Raman flow-cell.

In ATPE, raw FTIR data of light phase after ATPE reveal less obvious deviations when compared to Raman raw data. It is, however, important to note that the differences that are clearly visible in raw Raman data are only caused by the strong baseline increase due to underlying fluorescence. Indeed, the deviations in-between the FTIR spectra are less pronounced compared to Raman spectra. PLSR coefficients for mAb, HMW, and LMW are generally lower compared to the other methods. A possible explanation for this is that while amide I band can be identified in the FTIR spectra, amide II, and amide III bands are far less pronounced. Hence, the spectral information provides less distinguishable contributions by $\mathrm{mAb}$, and all other species in the multicomponent mixture. Since PLSR-model building relies on these different contributions to the fingerprint of the multicomponent matrix, regression becomes less efficient. The regression coefficients in ATPE were 0.94, 0.94, 0.98, with RMSEs of $0.167,0.013$, and $0.5 \mathrm{~g} / \mathrm{L}$, which is 9,8 , and $3.5 \%$ of the final mAb, HMW, and LMW concentrations, respectively.

After precipitation, larger deviations occur between wavenumbers 1800 and $1400 \mathrm{~cm}^{-1}$ for dissolution. No further preprocessing is necessary in case of FTIR data in dissolution. PLSR of FTIR-spectra of precipitation resulted in an $\mathrm{R}^{2}$ of 0.94 and RMSE of $0.43 \mathrm{~g} / \mathrm{L}$, referring to LMWs. In dissolution, the mAb concentration could be predicted with an $\mathrm{R}^{2}$ of 0.91 and a RMSE of $0.03 \mathrm{~g} / \mathrm{L}$. These deviations are equivalent to $4.2 \%$ for LMWs in precipitation and $2.8 \%$ for the final $\mathrm{mAb}$ concentration in dissolution.

For chromatography, spectra were shortened to the fingerprint region. The PLSR modeling for IgG resulted in an $\mathrm{R}^{2}$ of 0.92 including validation samples. RMSE of the target component was $0.24 \mathrm{~g} / \mathrm{L}$ or $4 \%$ of the highest sample measured. Figure 91 shows the regression results. In comparison to DAD and fluorescence the salt gradient shows a high influence on FTIR spectra, even in the shortened product-specific region. This is especially apparent between the wavenumbers $1700-1600 \mathrm{~cm}^{-1}$ and $1000-800 \mathrm{~cm}^{-1}$. The drift between $1000-800 \mathrm{~cm}^{-1}$ results mainly from the higher salt concentration, as there is a shift from higher product concentrations (blue) to lower product concentrations (red), see Figure 9k. Between $1700-1600 \mathrm{~cm}^{-1}$ the increase is mainly due to higher IgG concentration, as the high salt concentrations do not contribute to a rise in intensity. Best fit was achieved using three principal components in the PLSR model.

In UF/DF first derivatives of the raw FTIR spectra were calculated, as additive effects were the dominant type of effect. PLSR of the processed spectra yielded a regression coefficient of 0.95 with an RMSE of $1.09 \mathrm{~g} / \mathrm{L}$, which is equivalent to $5.5 \%$ of the final mAb concentration. 


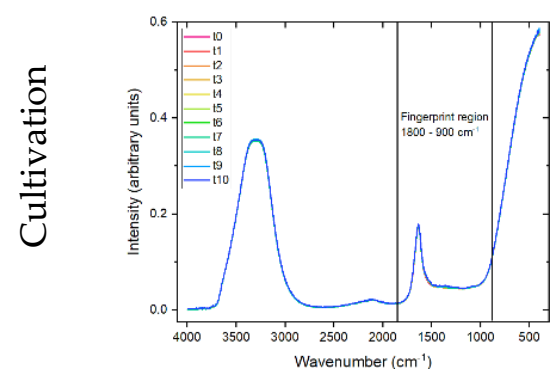

(a)

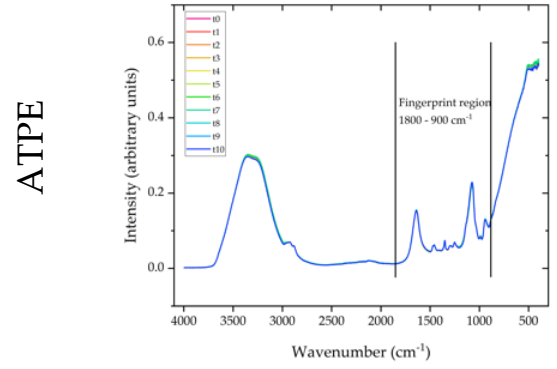

(d)

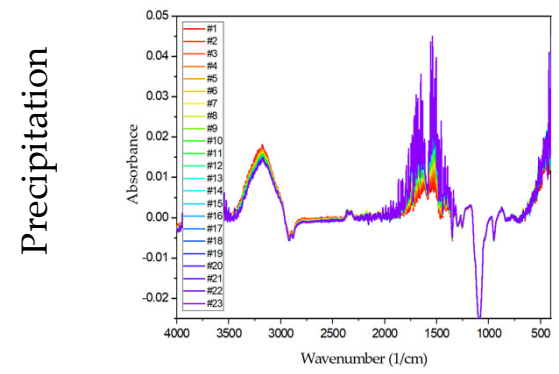

(g)

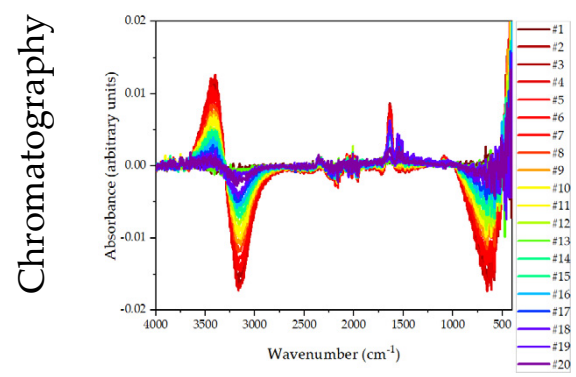

(j)

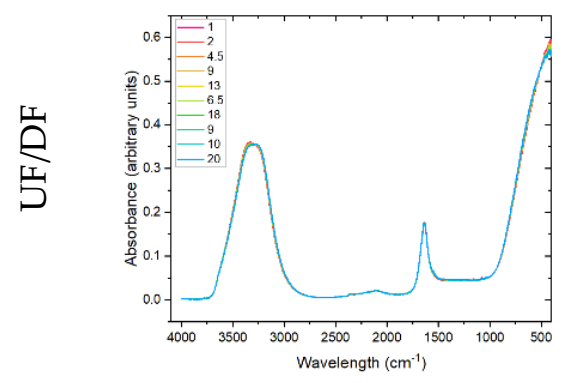

(m)

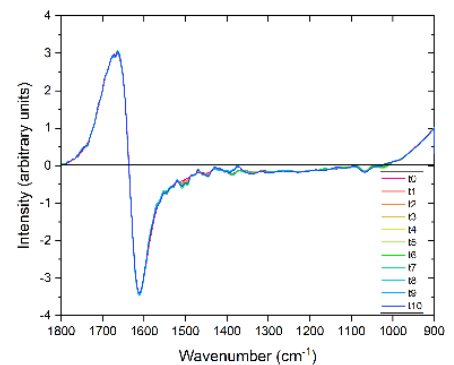

(b)

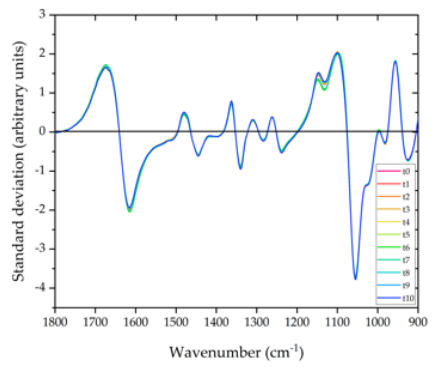

(e)

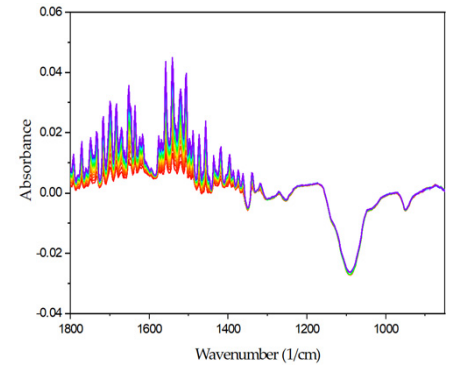

(h)

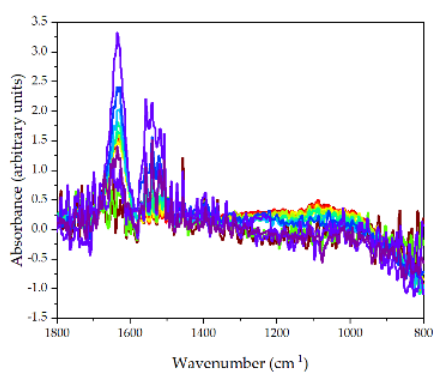

(k)

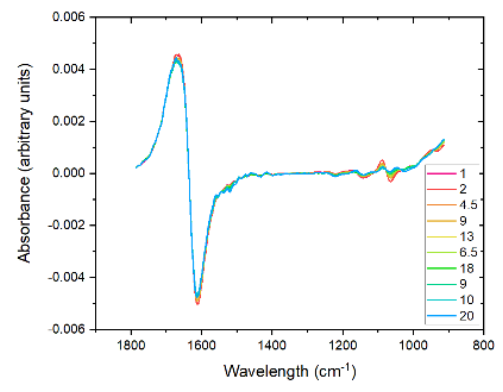

(n)

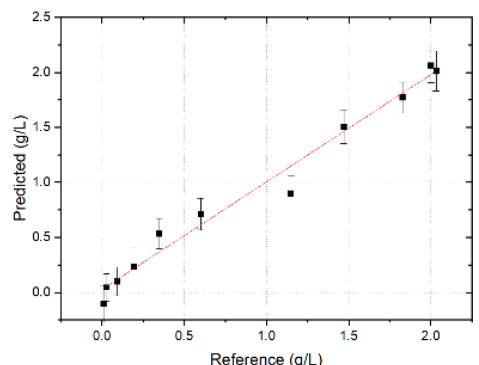

(c)

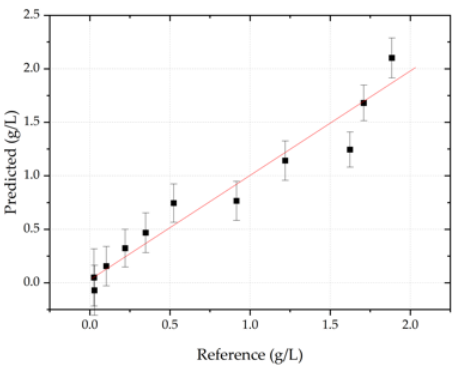

(f)

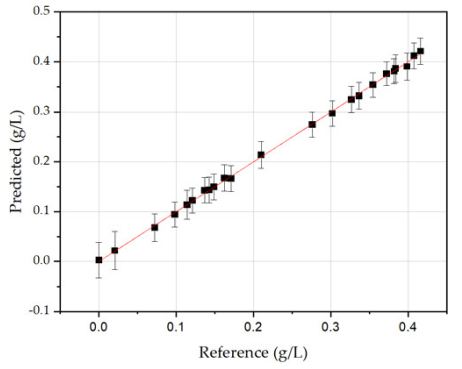

(i)

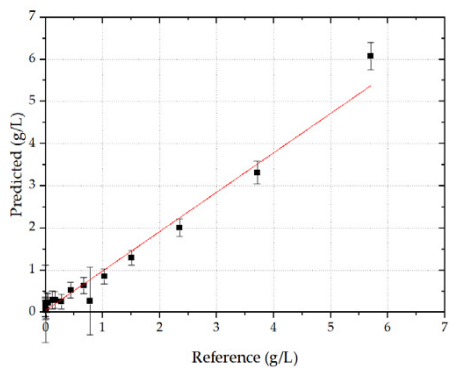

(1)

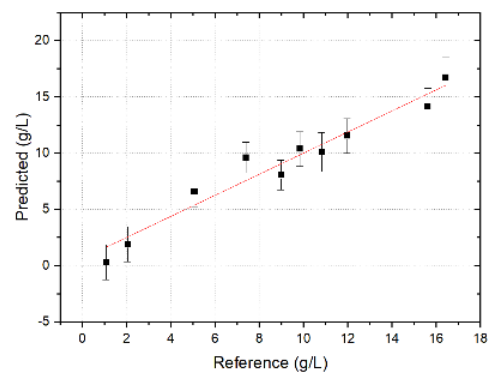

(o)

Figure 9. FTIR spectra: raw $(\mathbf{a}, \mathbf{d}, \mathbf{g}, \mathbf{j}, \mathbf{m})$, after preprocessing $(\mathbf{b}, \mathbf{e}, \mathbf{h}, \mathbf{k}, \mathbf{n})$ and PLSR results $(\mathbf{c}, \mathbf{f}, \mathbf{i}, \mathbf{l}, \mathbf{o})$. Spectra are color-coded from high concentration (blue) to low concentration (red). 


\subsection{Diode-Array Detector}

UV-Vis analytics rely on the principle of Lambert-Beer, as the spectrum measured by a DAD shows the absorption of monochromatic light by the analyte matrix. So, if there is only a single species present, or several species with non-overlapping absorption spectra, species concentrations can efficiently be correlated to the measured absorption at their specific absorption maximum. However, in multicomponent mixtures, such as the light phase in ATPE after processing HCCF, there is a significant overlap of all the different species-specific absorption characteristics. Applying the same chemometric methods that are common for Raman and FTIR analysis aims to extract the composition-specific UV-Vis spectral information. DAD spectra were measured in the range of $190-520 \mathrm{~nm}$. Since the method is based on absorption of UV-Vis light, increasing overall concentration leads to increasing absorption. These additive effects are removed by taking the first derivative of the spectra as described above. The derived spectra show that the strongest change in absorption is around $350 \mathrm{~nm}$. The regression coefficients of the prediction were $0.994,0.983$, and 0.994 , and RMSEs of $0.059 \mathrm{~g} / \mathrm{L}, 0.050 \mathrm{~g} / \mathrm{L}$, and $0.263 \mathrm{~g} / \mathrm{L}$, or $3 \%, 4.7 \%$, and $1.2 \%$ of the final concentration, for mAb, HMW, and LMW, respectively. It must be noted however that this region is not unambiguously assignable to protein or DNA, as these biomolecules have an absorption maximum at $280 \mathrm{~nm}$ and $260 \mathrm{~nm}$, respectively. It cannot be ruled out that the change in absorption may correlate to the color of the medium, which changes due to the addition of the two feed-media, which are red and green respectively. Moreover, polyaromatic substances like vitamins may absorb in this region. Further validation experiments are needed to validate the DAD predictions.

In ATPE, DAD obtained spectra reveal that with progressing cultivation time the biggest changes to the UV-Vis spectrum are around $280 \mathrm{~nm}$ wavelength. In the range of $320-520 \mathrm{~nm}$ there are additional smaller pronounced deviations to the spectrum. By using these processed spectra as predictors for the PLSR-model, regression coefficients of $0.99(\mathrm{mAb}), 0.80(\mathrm{HMW})$, and 0.95 (LMW) are obtained. The less efficient regression for HMW species is similar to the results in FTIR explainable by their small concentration and, therefore, smaller contribution to the extractable information in the UV-Vis spectrum. RMSE values were $0.027 \mathrm{~g} / \mathrm{L}, 0.011 \mathrm{~g} / \mathrm{L}$ and $0.22 \mathrm{~g} / \mathrm{L}$, or $1.4 \%, 6.7 \%$, and $1.5 \%$ of the final $\mathrm{mAb}, \mathrm{HMW}$, and LMW concentrations, respectively.

In precipitation, spectral data for the DAD are not shortened and the full spectrum is shown ranging from $190 \mathrm{~nm}$ to $520 \mathrm{~nm}$. The DAD is capable of detecting proteins reliably, but the distinction between different proteins is difficult. A similar trend can be observed in DAD raw data for dissolution. This is to be expected as the absorbance of a DAD increases according to the law of Lambert-Beer. DAD-PLSR regression for precipitation is very good $\left(\mathrm{R}^{2}\right.$ of 0.97 and RMSE of 0.26 ) referring to LMWs. In dissolution, convincing results are achieved ( $\mathrm{R}^{2}$ of 0.93 and RMSE of 0.02 ), but for the target component. These deviations are equivalent to $2.3 \%$ for LMWs in precipitation and $2.8 \%$ for the final $\mathrm{mAb}$ concentration in dissolution. It can be concluded that DAD is a suitable detector during precipitation for LMWs and in dissolution for the target component.

In chromatography, DAD spectra were shortened to $200-300 \mathrm{~nm}$. As this is the only part of the spectrum were significant absorption can be observed, see Figure 10j. Any preprocessing significantly worsened PLSR regression, eliminating effects the PLSR model can explain with the given concentrations. For the shortened DAD spectra, an $R^{2}$ of 0.94 for the IgG monomer was achieved in chromatography. RMSE for the DAD-PLSR was 0.021 or $7.6 \%$ of the highest sample measured. The regression results are illustrated in Figure 10. In the regression plot (l) a high variance for the zero-points is appeared. This results from the similar spectra (k). Between 200 and $225 \mathrm{~nm}$ the side components show a strong absorbance like the IgG monomer, the specificity of DAD-PLSR results from the stronger absorbance around $275 \mathrm{~nm}$. While the PLSR model can eliminate these overlapping effects to some degree, it is not possible to eliminate this overlapping completely. The best fit was achieved using six factors in the PLSR model. 


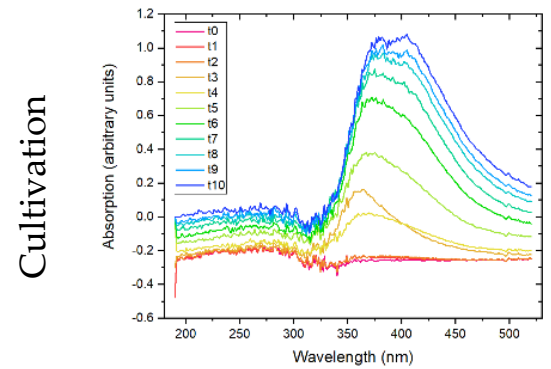

(a)

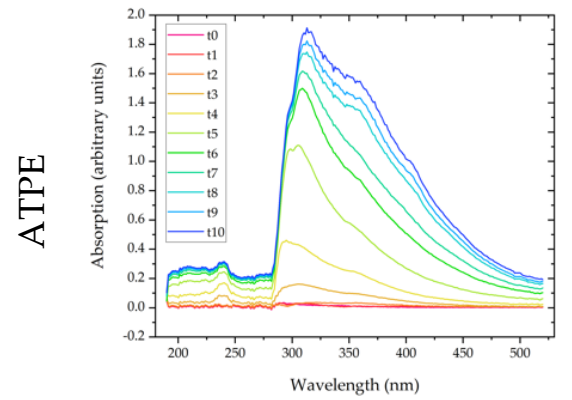

(d)

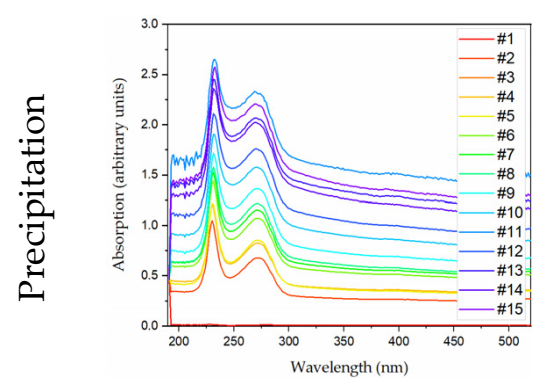

(g)

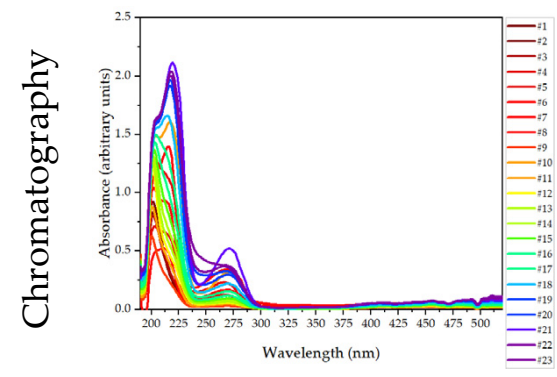

(j)

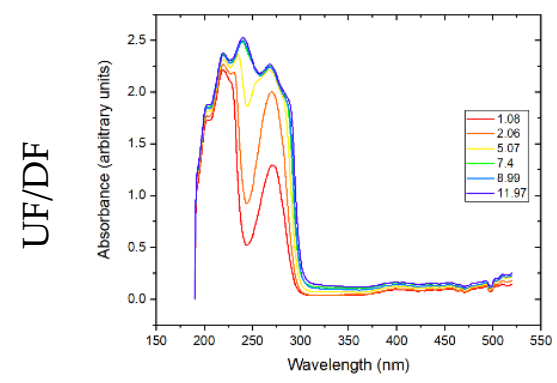

(m)

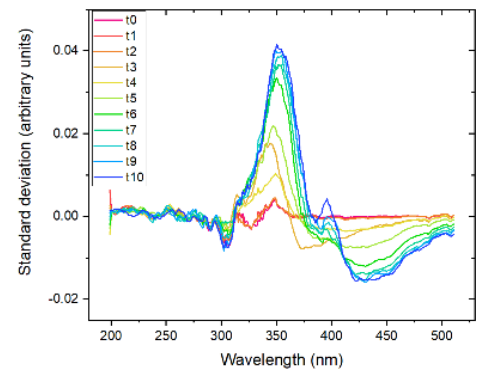

(b)

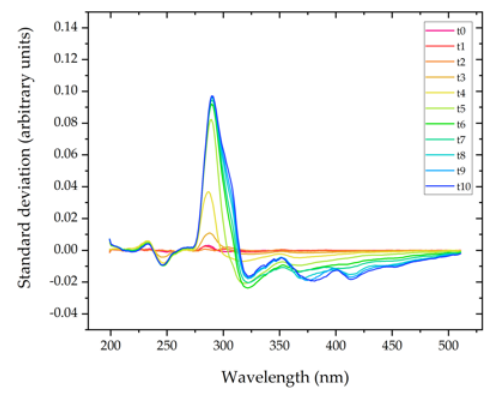

(e)

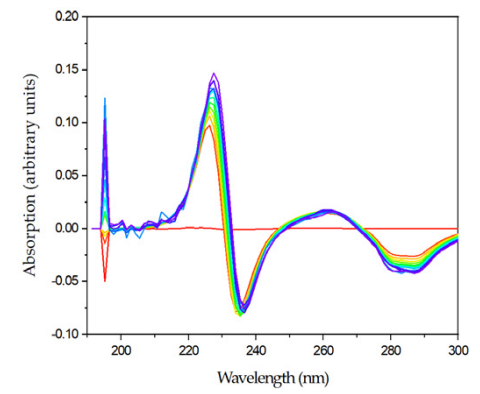

(h)

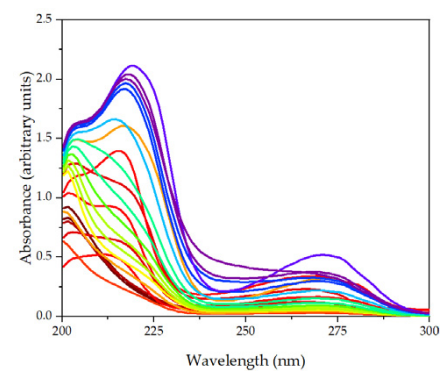

(k)

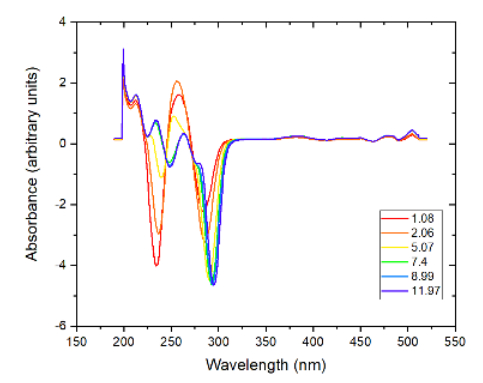

(n)

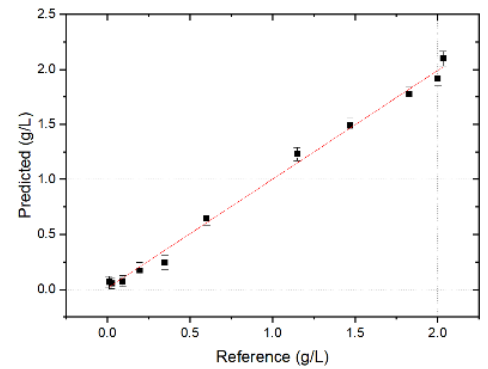

(c)

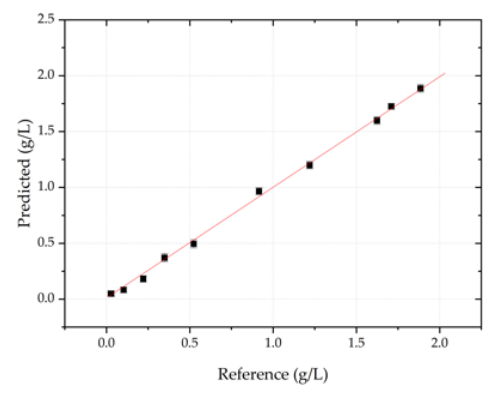

(f)

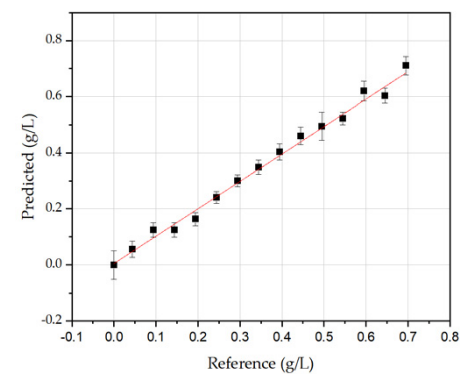

(i)

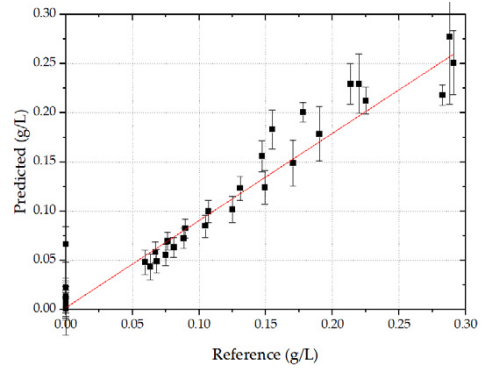

(1)

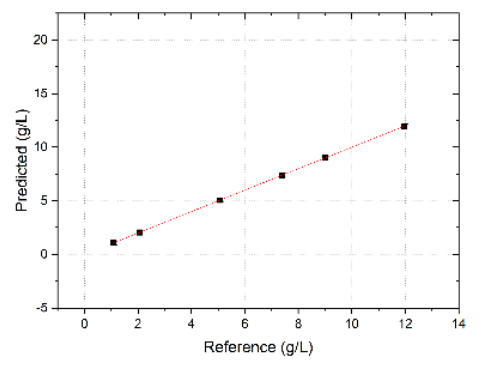

(o)

Figure 10. DAD spectra: raw $(\mathbf{a}, \mathbf{d}, \mathbf{g}, \mathbf{j}, \mathbf{m})$, after preprocessing $(\mathbf{b}, \mathbf{e}, \mathbf{h}, \mathbf{k}, \mathbf{n})$ and PLSR results $(\mathbf{c}, \mathbf{f}, \mathbf{i}, \mathbf{l}, \mathbf{o})$. Spectra are color-coded from high concentration (blue) to low concentration (red). 
In UF/DF for the DAD, primarily additive effects were observed in the raw spectra. Thus, first derivatives of the raw spectra were calculated and PLSR was performed. An excellent regression coefficient of $>0.999$ with a RMSE of $0.005 \mathrm{~g} / \mathrm{L}$ was found for the prediction of the $\mathrm{mAb}$ concentration, which is $0.03 \%$ of the final $\mathrm{mAb}$ concentration.

\subsection{Fluorescence Emission Spectra}

Like UV-Vis measurements, fluorescence spectra are based on the absorption of photons. Therefore, also in fluorescence measurements strong additive effects were observed in USP, which were removed by calculating first derivatives of emission spectra. In the present experimental setup, the samples were excited at $280 \mathrm{~nm}$, which is the absorption maximum of tryptophan. The emission spectrum was recorded from 280 to $900 \mathrm{~nm}$. First derivatives revealed that strong changes in fluorescence intensity are observed at $340-360 \mathrm{~nm}$, which is the emission maximum for tryptophan, i.e., protein. Regression coefficients for mAb, HMW, and LMW were $0.955,0.892,0.955$, with RMSEs of $0.166 \mathrm{~g} / \mathrm{L}, 0.126 \mathrm{~g} / \mathrm{L}$ and $0.717 \mathrm{~g} / \mathrm{L}$, or $8.3 \%, 11.8 \%$, and $3.3 \%$ of the final concentration, respectively.

In ATPE, the spectra show the expected emission maximum of proteins at $350 \mathrm{~nm}$. A second major peak can be seen $550 \mathrm{~nm}$. Preprocessing included first derivative and SNV normalization. Regression coefficients of $0.85(\mathrm{mAb}), 0.74$ (HMW), and 0.85 (LMW) are obtained by PLSR. The corresponding RMSE values were $0.128 \mathrm{~g} / \mathrm{L}, 0.018 \mathrm{~g} / \mathrm{L}$, and $0.542 \mathrm{~g} / \mathrm{L}$, which is $6.8 \%, 10.9 \%$, and $3.8 \%$ of the final mAb, HMW, and LMW concentrations, respectively.

In precipitation, the entire spectrum was used for analysis. Analyzing the raw data an absorption maximum between 570 and $675 \mathrm{~nm}$ can be identified, which describes the concentration increase during dissolution satisfactory. PLSR analysis resulted in an $\mathrm{R}^{2}$ of 0.90 with a RMSE of 0.02 . These deviations are equivalent to $3.8 \%$ for the final $\mathrm{mAb}$ concentration in dissolution. The regression is depicted in Figure 11.

In chromatography, fluorescence spectra were shortened to $580-700 \mathrm{~nm}$. Fluorescence data were not preprocessed, which would eliminate some of the information contained in the spectra, worsening PLSR. For fluorescence the $\mathrm{R}^{2}$ was 0.93 . The RMSE was 0.024 or $7.9 \%$ of the highest sample. The regression results are depicted in Figure 11. In comparison to DAD, see Figure 10. The fluorescence model predicts low concentration samples more accurately. This can be explained interpreting the spectra, see (b). The samples containing high concentrations of IgG emit most light at around $660 \mathrm{~nm}$, while for low IgG concentration samples and high side concentration samples the fluorescence maximum is shifted to $680 \mathrm{~nm}$ and $600 \mathrm{~nm}$ respectively.

In UF/DF, first derivatives were calculated from the raw emission spectra, which were then regressed using PLSR. A good regression coefficient of 0.986, with a RMSE of $0.705 \mathrm{~g} / \mathrm{L}$, was found. This corresponds to a deviation of $3.5 \%$ with respect to the final $\mathrm{mAb}$ concentration.

\subsection{Combination of Spectroscopic Data}

For the analysis of cell culture samples, Raman and FTIR spectroscopy showed the most promising results. Therefore, the combined analysis of Raman and fluorescence spectra in one PLSR was evaluated. The combination of Raman and FTIR data yielded similar regression coefficients as the two single methods of 0.991 for mAb, 0.995 for HMW and 0.996 for LMW, with RMSEs of $0.073,0.028$, and $0.207 \mathrm{~g} / \mathrm{L}, 3.7 \%, 2.6 \%$, and $1 \%$ of the final concentration, respectively. Although this might not seem advantageous at first, it must be considered that both methods yield different information, thereby providing potentially a more robust prediction.

Addition of DAD spectra further enhanced the overall regression as the regression coefficients increased to 0.996 (mAb), 0.993 (HMW), and 0.997 (LMW), with RMSEs of $0.048,0.032$, and $0.170 \mathrm{~g} / \mathrm{L}, 2.4 \%, 3 \%$, and $0.8 \%$ of the final concentration, respectively. This indicates, that further orthogonal measurement methods may further improve the overall predictive power of spectroscopic PAT-tools. 


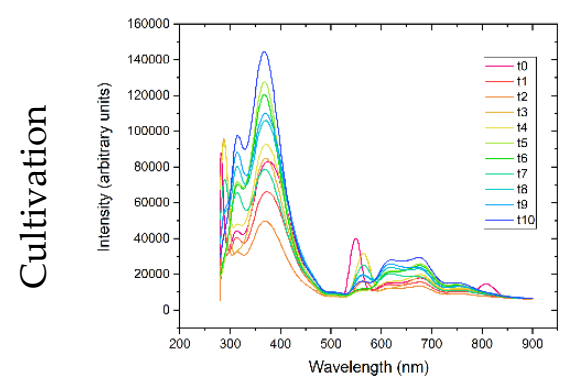

(a)

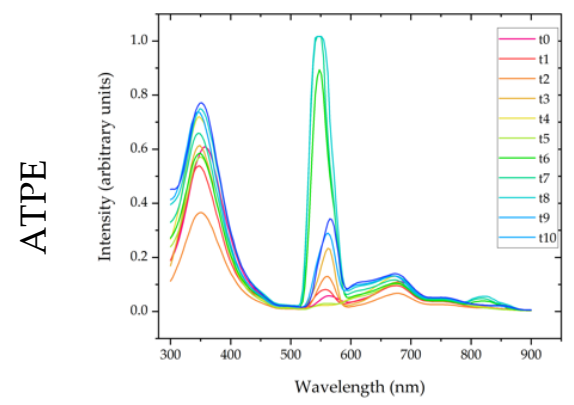

(d)

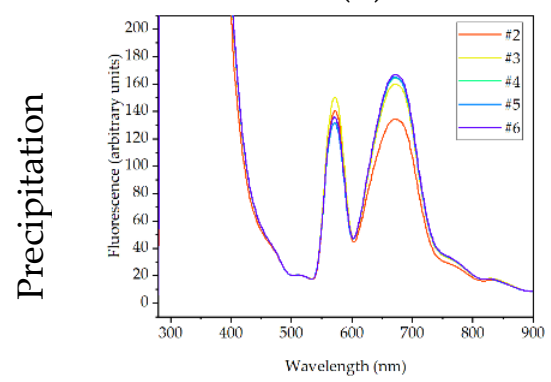

(g)

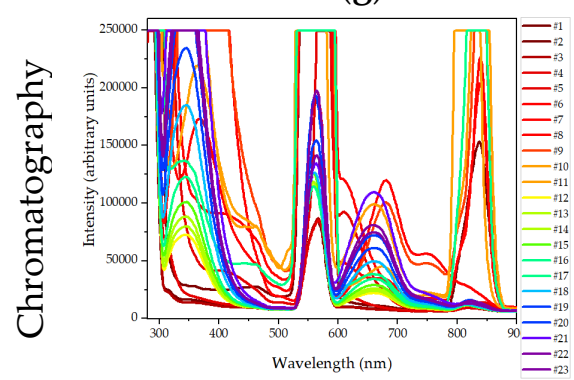

(j)

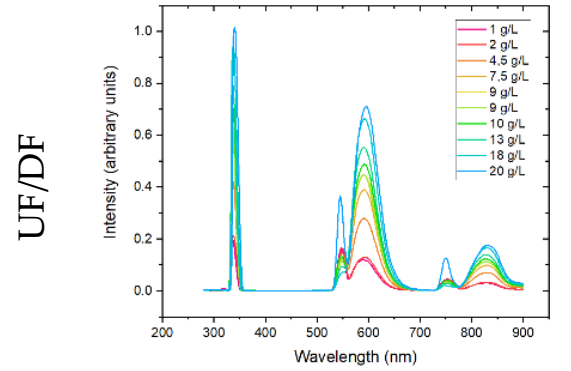

(m)

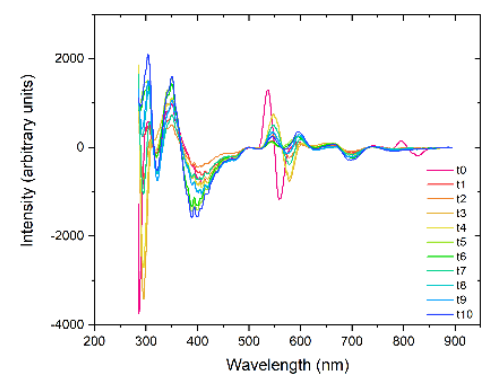

(b)

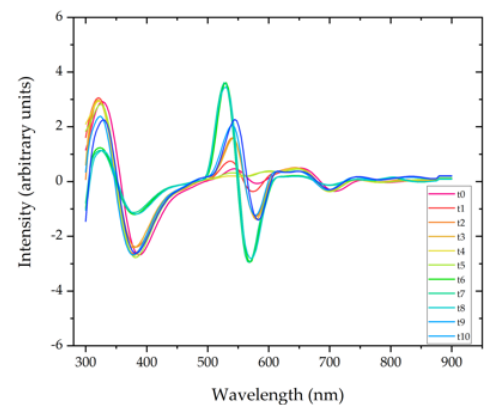

(e)

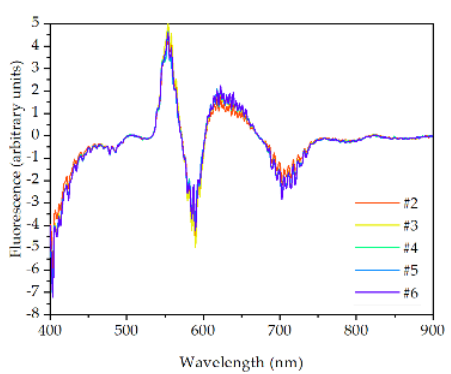

(h)

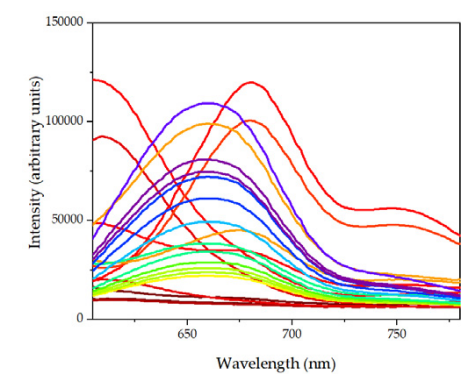

(k)

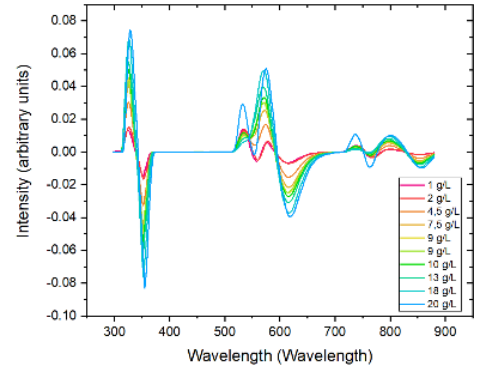

(n)

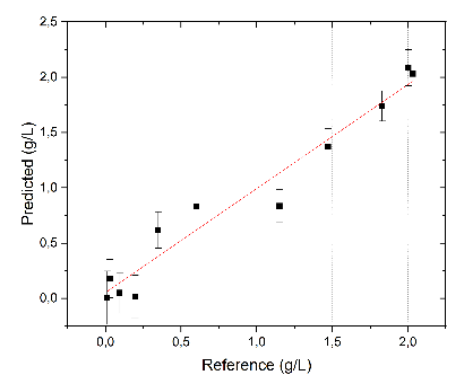

(c)

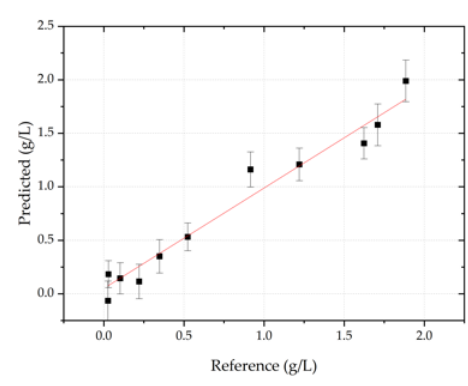

(f)

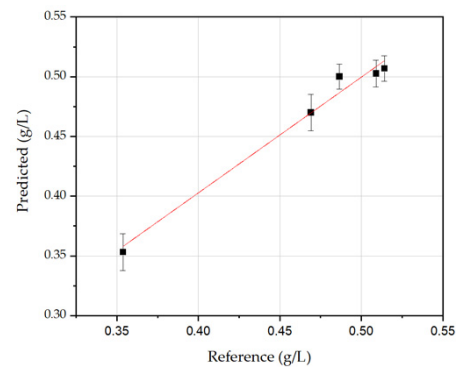

(i)

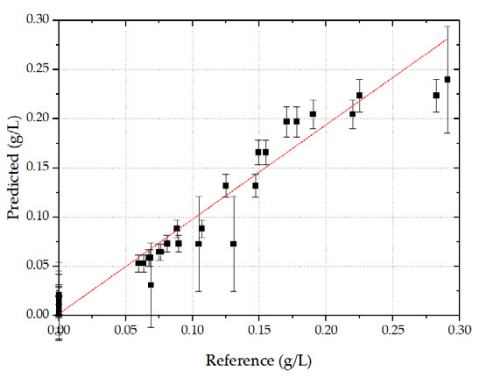

(1)

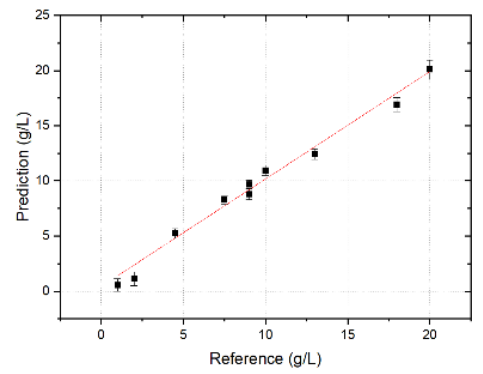

(o)

Figure 11. Fluorescence spectra: raw $(\mathbf{a}, \mathbf{d}, \mathbf{g}, \mathbf{j}, \mathbf{m})$, after preprocessing $(\mathbf{b}, \mathbf{e}, \mathbf{h}, \mathbf{k}, \mathbf{n})$ and PLSR results $(\mathbf{c}, \mathbf{f}, \mathbf{i}, \mathbf{l}, \mathbf{o})$. Spectra are color-coded from high concentration (blue) to low concentration (red). 
In ATPE, the same preprocessing procedure was applied. However, prior to PLSR combined spectra were normalized to their respective maximum to even the amplitudes. The combination of Raman and FTIR yielded regression coefficients of 0.97 (mAb), 0.85 (HMW), and 0.97 (LMW). By further expanding the range of orthogonal predictor data by adding DAD obtained spectra, regression coefficients of 0.99 (mAb), 0.87 (HMW), and 0.96 (LMW) are obtained. This simple approach of combining spectral data reveals that just combining the spectra as predictor set in PLSR does not yield a higher prediction accuracy. Rather it seems that the algorithm still favors the most suitable data, which, in case of ATPE, was Raman. Hence, the obtained regression coefficients are similar to the initial results by Raman alone. More sophisticated approaches in combining different data set, are needed to investigate possible benefits in accuracy. However, having orthogonal detector data at hand, can enhance the reliability of the overall control strategy.

For the precipitation unit, DAD and Raman have each given the best performance in dissolution with an $\mathrm{R}^{2}$ of 0.93 (mAb). Raman correlated to purity with an $\mathrm{R}^{2}$ of 0.85 . Prediction of purity was not possible with DAD alone. Therefore, the combination of the two sensors in one PLSR is investigated. Both detectors in combination achieved an $R^{2}$ of 0.90 for prediction of the target component and resulted in an $R^{2}$ of 0.72 for purity. Although the regression coefficient for the combination of detectors is lower than for the single detectors, the orthogonal nature of both detectors increases the robustness.

In chromatography, a combination of DAD and fluorescence data showed significant improvement in prediction quality. For IgG concentration an $\mathrm{R}^{2}$ of 0.93 was achieved. In addition, the low prediction error for spectroscopic data with no IgG content, which was observed in fluorescence data but not in DAD data, was reproduced. For LMW 1 , an $R^{2}$ of 0.91; for LMW 2, an $R^{2}$ of 0.93 was achieved. $R^{2}$ for the IgG dimer is 0.67 and could not be improved. The reason for this might be the low concentration of $0.022 \mathrm{~g} / \mathrm{L}$ at maximum, and, therefore, the system-immanent error resulting from offline analytics methodology. RMSE for the combined PLSR model was 0.027 for IgG, 0.0047 for dimer, 0.016 for LMW 1, and 0.014 for LMW 2 which corresponds to $9.7 \%, 20 \%, 13.9 \%$ and $10 \%$ respectively.

\subsection{Additional Process Data}

PAT may not only cover spectroscopic measurements but also include all other available online data sources for providing means of achieving APC. In cell culture these include measurement of $\mathrm{pH}$ and $\mathrm{pO}_{2}$, but also conductivity [5], $\mathrm{pCO}_{2}$, off-gas analysis and turbidity. Changes in $\mathrm{pH}, \mathrm{pO}_{2}, \mathrm{pCO}_{2}$, off-gas composition can be used to predict the metabolic state of the cells by means of oxygen consumption and carbon dioxide production. $\mathrm{CHO}$ cells typically exhibit overflow metabolism that is characterized by high rates of lactate excretion. Hence, most carbon from glucose is not initially converted to $\mathrm{CO}_{2}$. Switch to an oxidative metabolic phenotype might be detected by altered patterns of oxygen consumption and $\mathrm{CO}_{2}$ excretion, which would also decrease the $\mathrm{pH}$. While turbidity yields information about the total cell concentration, conductivity changes can be correlated to decreasing viability, whereby the combination of both methods can be used to predict viable cell concentration [5].

In ATPE, the $\mathrm{pH}(6.4 \pm 0.1)$, conductivity $(12 \pm 2 \mathrm{mS} / \mathrm{cm})$ and density $\left(1104 \pm 4 \mathrm{~kg} / \mathrm{m}^{3}\right)$ strongly depend on the properties of the phase forming components, in this case PEG400 and phosphate salt. Since the properties of polymer and salt buffers do not change during the process, no correlation between species concentrations and other sensor data were found. Turbidity ( $37.5 \pm 12.5 \mathrm{FAU})$ is constantly low. However, it is important to keep in mind that these data still hold value and should be continuously monitored, as they are suitable to check if the process operates within specifications as part of a control strategy.

In precipitation, the $\mathrm{pH}$ value remains constant throughout precipitation which indicates that no process information can be correlated to this sensor signal. The conductivity $(12 \mathrm{mS} / \mathrm{cm})$ is depend on the light phase which contains residual phosphate salt as described in the ATPE section. Since the added PEG solution is not conductive after PEG addition the conductivity decreases. The resulting conductivity change can be 
correlated to the composition of the dispersion and can be used as control PAT. Turbidity has, like conductivity, a strong deflection during addition of PEG because precipitate formation occurs instantaneously. Turbidity is not specific to one protein, but can be used as control sensor to monitor precipitation progress. During dissolution, the $\mathrm{pH}$ value $(\mathrm{pH}=5.9 \pm 0.1)$, conductivity $(1.5 \pm 0.1 \mathrm{mS} / \mathrm{cm})$ and turbidity $(0 \mathrm{FAU})$ remained constant during redissolution of precipitates.

During chromatography, $\mathrm{pH}$ and conductivity were measured. $\mathrm{pH}$ maintains constant during chromatography, with a minor change of \pm 0.02 during side and target component elution at 23 to $30 \mathrm{~min}$. The course of conductivity during chromatography is mainly dominated by the changing salt concentration. While the elution time of the components can be detected using $\mathrm{pH}$, a significant change in the determination coefficient of the PLSR model was not observed. However, especially $\mathrm{pH}$ and conductivity changes are especially known to be valid and of substantial aid for process operation performance and maintenance prediction systems [75].

In UF/DF, the salt concentration can be correlated to conductivity. $\mathrm{pH}$ can be correlated to progress of buffer exchange. Turbidity is not applicable anymore such late in the process.

\subsection{Analytics \\ 3.7.1. ELISA}

The most critical quality attribute is the biological activity of the therapeutic protein, as this determines whether it retains its efficacy. For this reason, a specific direct enzymelinked immunosorbent assay (ELISA) was performed to examine the preservation of biological activity after each unit operation. In Figure 17 the activity of detected mAb is shown. It can be seen that biological activity is successfully maintained after ATPE, precipitation and chromatography.

\subsubsection{Bradford}

The Bradford test is a colorimetric method for determination of total protein concentration. The test is based on the binding of Coomassie Brilliant Blue G-250 to acidic amino acid residues. Upon binding of the dye to the amino acids under acidic conditions, a shift of the dye's absorption maximum occurs, which causes a color change from red/brown to blue. This color shift is proportional to the total protein concentration, though care must be taken as different protein compositions can cause differences in dye binding intensity and accordingly color change. Therefore, the choice of a proper, representative, protein for generating a calibration curve is very important. Figure 17 shows the results for total protein concentration determination.

\subsubsection{SDS-PAGE}

The 2D Gel electrophoresis is used as a visualizing method for detection of proteins. In the first dimension, the proteins are focused according to their isoelectric point in a $\mathrm{pH}$ gradient (pH 3-10). Following, they are separated according to their size through gel electrophoresis. Heavy chain (HC) and light chain (LC) of the mAb can be clearly identified. Reduction of protein concentration throughout the process can be observed. The highest side component concentrations are found in cultivation broth (top right). Gels are depicted in Figure 12.

\subsubsection{DNA Concentration Determination}

The concentration of double stranded (ds)DNA can be determined using intercalating dyes such as SYBR Green. The DNA-dye complex is excited at $480 \mathrm{~nm}$ and the emission is measured at $520 \mathrm{~nm}$. The fluorescence intensity is proportional to the amount of dsDNA and the concentration can be determined by comparison to a fluorescence signal of a sample with known DNA concentration. Figure 17 shows the results for dsDNA concentration determination. It can be seen that ATPE achieves a 90\% reduction in DNA concentration and the remaining DNA is removed in precipitation. 


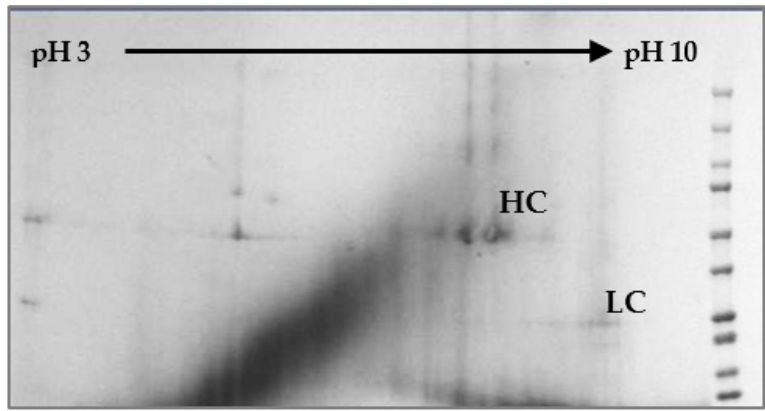

(a)

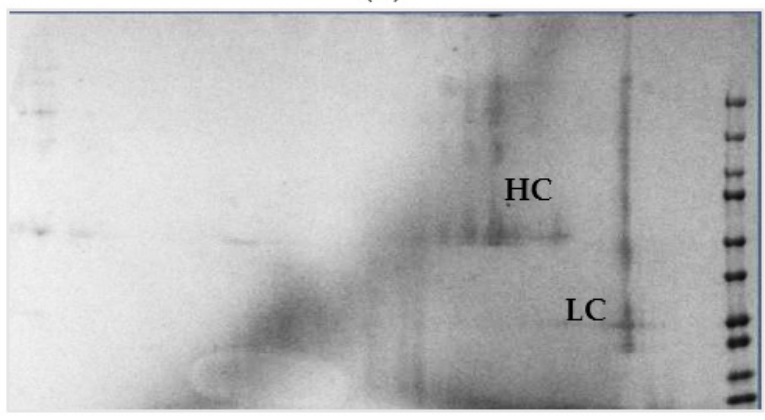

(c)

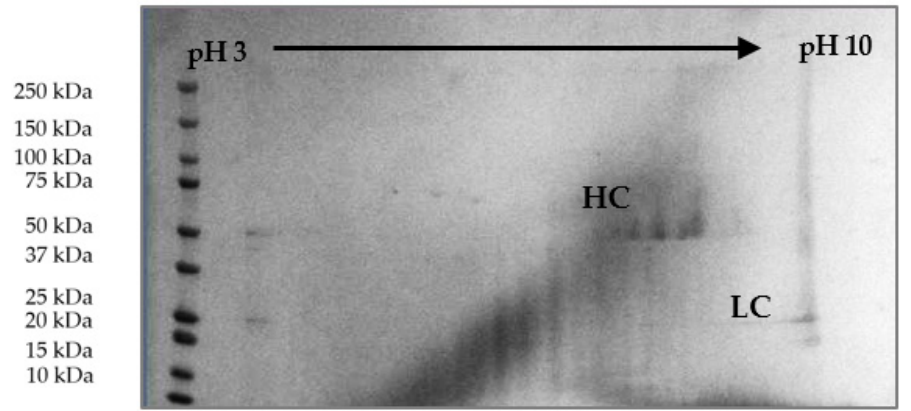

(b)

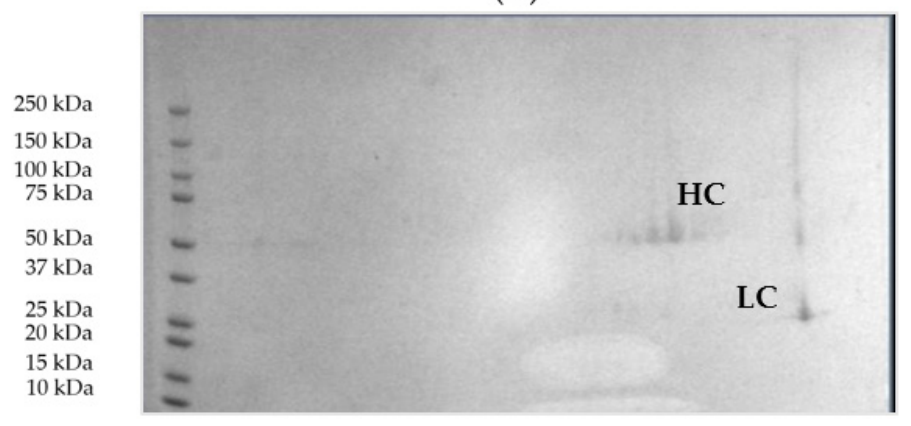

(d)

Figure 12. Two-dimensional (2D) SDS-PAGE gels of (a) light phase after ATPE, (b) cultivation broth, (c) dissolved mAb after precipiation, (d) mAb fraction after chromatography. LC: light chain of mAb. HC: heavy chain of mAb.

\subsection{Process Simulation Studies to Propose Advanced Process Control Concepts}

In literature concentration fluctuations or a continuous $\mathrm{mAb}$ concentration decrease due to decreasing cell specific productivity over process time have been described [6]. Simulation studies are used to test whether PAT can enable a process control strategy that allows for compensation of titer fluctuations in the subsequent DSP. The scenario for the following simulations is that the mAb concentration and purity (i.e., HMW and LMW concentrations) are continuously measured in the outstream of each unit operation using the detector array presented above. The real time measurement data are continuously forwarded to the following unit operation in the $\mathrm{mAb}$ manufacturing process. This information is fed into a process model that calculates the necessary process adjustment to reach either a constant $\mathrm{mAb}$ concentration or constant volume flow.

Since the focus of the study presented here is not just simply the process models, but the application and demonstration, in combination with the already discussed spectroscopybased PAT, we refer here again to the publications on the respective unit operations.

Simulation of viable cell concentration and product concentration in perfusion mode is shown in Figure 13. The viable cell and product concentration increases over the first $72 \mathrm{~h}$. Then the perfusion is started and the product concentration decreases momentarily since more product is washed out than is produced. Shortly after the concentration increases again proportionally to the viable cell concentration until the steady state is reached after approximately $350 \mathrm{~h}$. Product concentration in the steady state reaches $1.8 \mathrm{~g} / \mathrm{L}$. As a basis for the following unit operations, a final steady state concentration of $2 \mathrm{~g} / \mathrm{L}$ is assumed.

Figure 14 shows how concentration fluctuations in USP are processed to a constant concentration in the product containing light phase. Polymer and salt solutions are mixed based on the phase equilibrium within the same tie-line. Based on the lever-arm rule, either less or more light phase is produced, thereby concentration is kept constant. Operating on the same tie-line is necessary to ensure constant yield and product phase properties. 


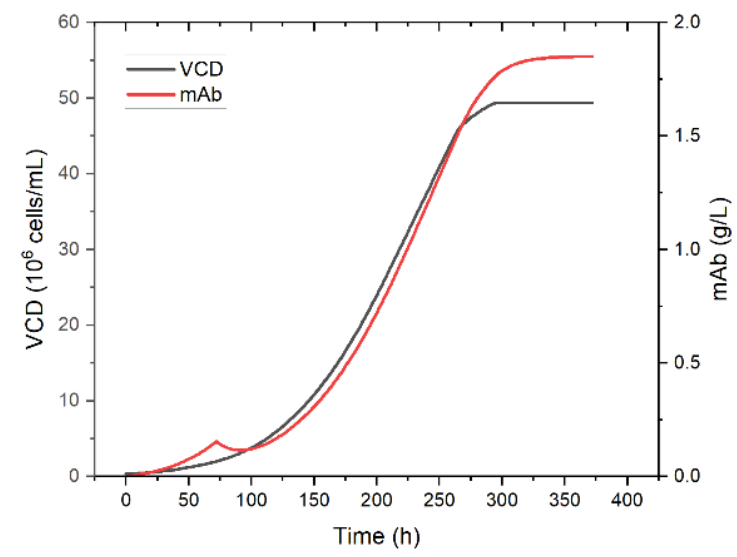

Figure 13. Simulation results of viable cell and product concentration in perfusion.

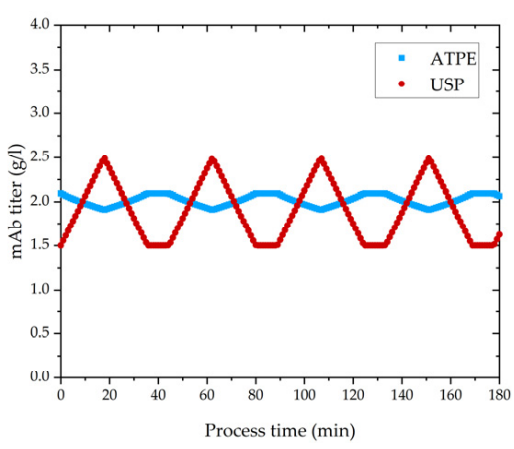

(a)

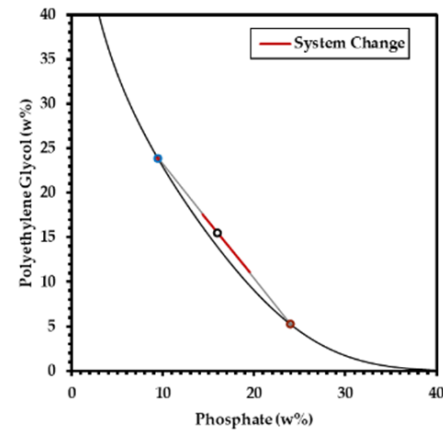

(b)

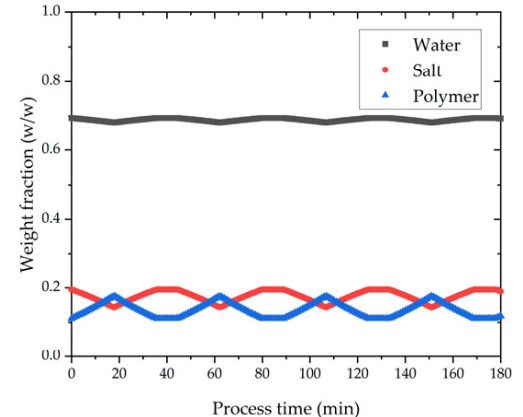

(c)

Figure 14. Simulation results for concentration fluctuations (a) and concentration compensation by system adjustment (b,c).

In Figure 15, the concentration change in precipitation over process time is depicted. Precipitation is a concentration independent process [73], which can be also verified with these results. The ratio of PEG solution and light phase do not change with varying $\mathrm{mAb}$ concentration because the PEG content is calculated based on the volume coming from extraction and not respectively to the concentration. In this way concentration variations do not affect precipitation. For higher titers precipitation takes slightly longer (few seconds) as show in Figure 15, but the result is the same, and lead to complete precipitation. Complete precipitation is a function of mixing of light phase and PEG solution.

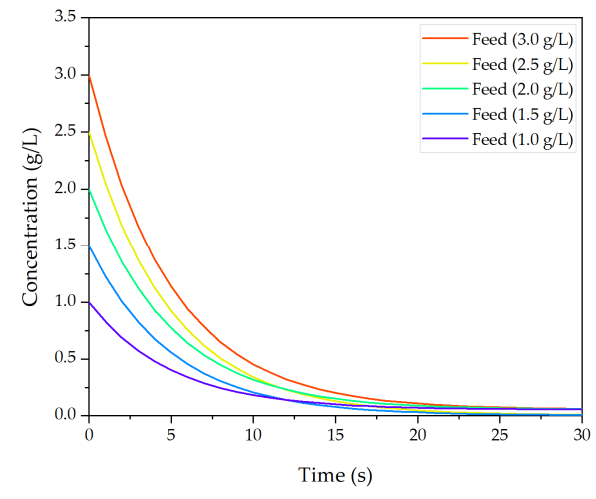

(a)

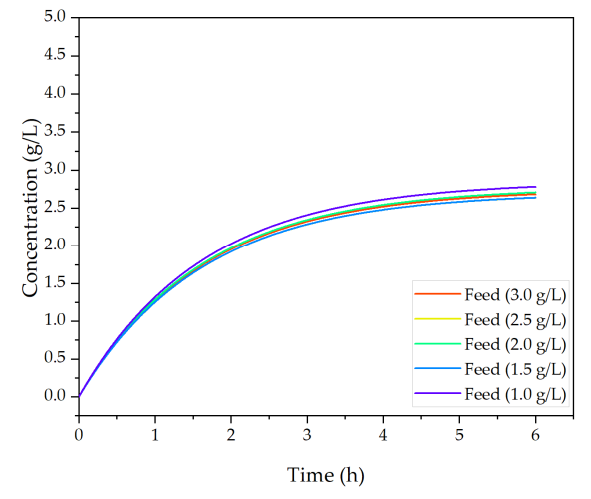

(b)

Figure 15. Simulation results for concentration fluctuations (a) for precipitation and (b) dissolution. 
In contrast, dissolution is concentration dependent and able to react to decreased or increased feed concentration by adjusting the dissolution ratio. In this manner the precipitation unit can provide a constant concentration for chromatography, which is shown in Figure 15b. For different feed concentrations, the dilution ratio was adjusted resulting in a constant output concentration of $2.6 \mathrm{~g} / \mathrm{L}( \pm 0.05)$. Changes in flow rate can be compensated with a higher stream of PEG solution to keep precipitations condition constant and ensure complete precipitation. Due to the fact that dead-end filtration eliminates the complete volume of supernatant around precipitates, flow rate fluctuation does not affect dissolution. Hence, concentration and flow rate changes cannot be compensated at once since a constant $\mathrm{mAb}$ concentration is accompanied by a fluctuation in flow rate. Compensation of purity was not possible in precipitation.

In Figure 16, simulation results from integrated counter current chromatography (iCCC) modeling are illustrated. In (a), the chromatogram of ion exchange chromatography (IEX) is given. Between 400 and 1000 s, the majority of LMW 1 and LMW 2 are eluted. This is due to the loading of the strong-binding fraction of hydrophobic interaction chromatography (HIC) and the weak-binding fraction of IEX. IgG is eluted from 2250 to $2500 \mathrm{~s}$. Cutting points are marked with dashed lines and are set at $0.05 \mathrm{~g} / \mathrm{L}$ which was detectable in chromatography using the PAT system described above.

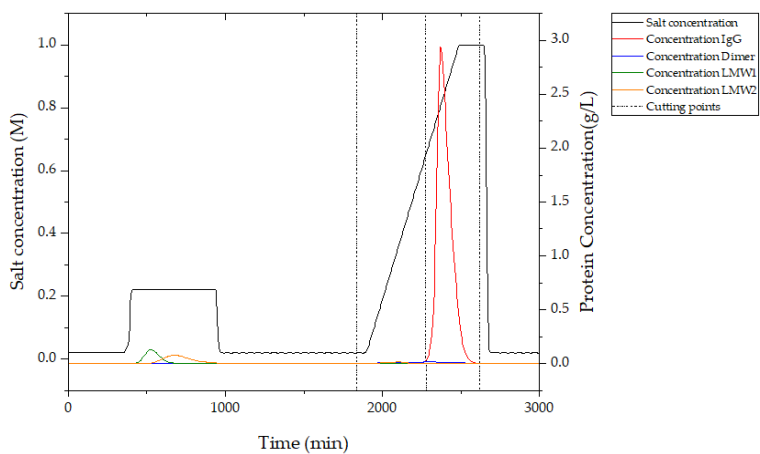

(a)

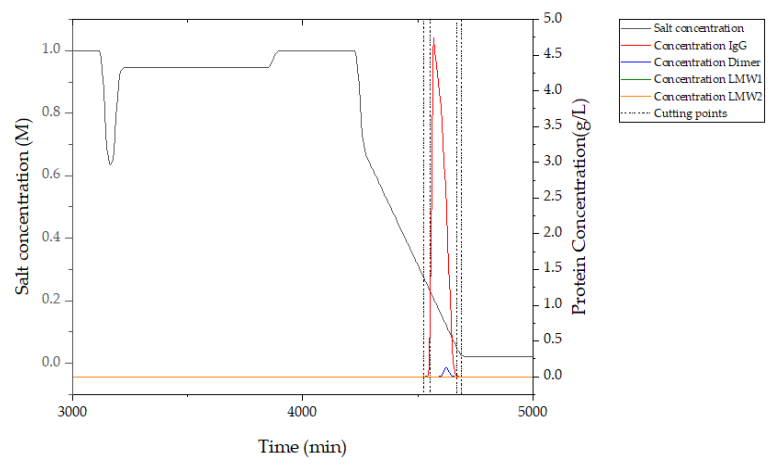

(b)

Figure 16. Simulation results of integrated counter current chromatography (iCCC); (a) is ion exchange chromatography (IEX) after five cycles, (b) is hydrophobic interaction chromatography (HIC) after five cycles.

This simulation shows that an in-line process control using a PAT system of DAD and/or fluorescence leads to a very high process yield, since cutting points in the chromatogram can be automatically detected, controlling the fractionation.

\section{Discussion}

\subsection{Applicability of Spectroscopic Methods in Continuous Biomanufacturing}

The evaluation of the different detector and sensor data in USP and ATPE reveals that Raman was not only the most reliable technique for mAb, but also for HMW and LMW prediction. It is also the most easily implementable spectroscopic technique as in-line probes and flow cells are widely available. Data acquisition is also sufficiently fast (few seconds) for USP and ATPE. FTIR data are also suitable for building a PLSR, however less reliable when compared to Raman. Due to the overall lesser observed variability in the spectra, it also appears to be less sensitive to changes in species concentration. DAD yielded very good correlation results, however, there are no in-line probes readily available and the detection of absorption is by principle less species-specific than Raman fingerprints and therefore more likely to give false-positively predictions. Fluorescence yielded the worst correlation results and is therefore less likely to be implemented as a primary detector technique in USP and ATPE. Overall, for in-line mAb, HMW and LMW analytics, Raman is the recommend spectroscopic technique. 
Simulation results showed that based on the continuous measurement of mAb, HMW and LMW concentrations by the PAT sensors, the unit operations in DSP could be controlled using process models that calculate necessary system adjustments in order to keep either concentration or volume flow constant.

For the precipitation unit, evaluation of Raman, FITR, DAD, and fluorescence yielded different results for precipitation and dissolution. During precipitation, experimental data show that the prediction of the target component and HMWs is poor. The results for HMWs are not surprising, since most of this impurity is already removed in ATPE. For LMWs very good results can be obtained with Raman $\left(R^{2}\right.$ of 0.95$)$, FTIR ( $R^{2}$ of 0.94$)$, and DAD ( ${ }^{2}$ of 0.97). Fluorescence could not be tested due to the precipitates which are harmful for the flow-through cell of the detector. Unfortunately, no satisfactory results for the prediction of purity was found with any of the detectors during precipitation. For dissolution, all four detectors could reliably predict the concentration of the target component. Nevertheless, only poor correlations were found for HMWs and LMWs since most impurities are already separated before dissolution is performed. Only Raman was capable to predict the purity of HMWs ( $R^{2}$ of 0.13 ), LMWs ( $R^{2}$ of 0.22$)$, and the target component $\left(R^{2}\right.$ of 0.85$)$, whereby, only the precision of the TC was convenient. Therefore, similar to USP and ATPE, Raman is the best suited sensor for the precipitation unit.

According to this study, Raman is recommended as a detector for precipitation since it has shown persuasive results in detecting the target and side components (LMWs) in precipitation and dissolution. Additionally, purity of the target component in the dissolution could be correlated with Raman. As an orthogonal measurement strategy, a DAD is recommended due to fast acquisition time and precision. Furthermore, a conductivity probe is recommended as PAT control strategy for detection of optimal conditions during precipitation.

In chromatography, advanced process control using in-line measurements is the most promising way to establish continuous downstream manufacturing in the purification of biopharmaceuticals. In highly purified solutions of $\mathrm{mAb}$, a way to detect impurities even at very low concentration is a combination of DAD and fluorescence. In this study, a concentration down to $0.05 \mathrm{~g} / \mathrm{L}$ could be measured using this combination. Using the combination of DAD and fluorescence, an $\mathrm{R}^{2}$ of 0.93 for the target component was achieved. Regression coefficients for HCP impurities were comparable, with 0.91 and 0.93 respectively. Regression coefficient for the dimer was 0.67 , which probably resulted from the low concentrations observed in chromatography, with around 0.005 to $0.025 \mathrm{~g} / \mathrm{L}$, which is very close to the detection limit of the employed SEC chromatography. In DAD measurements, without simultaneously evaluating fluorescence a high variation in zero concentration measurements for the product was observed. This would be a problem for inline product detection, with resulting background noise interfering with peak detection. Using a combination of DAD and fluorescence, this problem is eliminated. FTIR could be used in the present experimental setup, however, it has the downside of a lower sensitivity. Therefore, detecting incoming peaks is more difficult. Later detection, at higher concentrations would result in a lower process yield in preparative process. This, lower sensitivity observed in chromatography likely results in the overlapping absorbance ranges of the changing buffer solution and the target component, which is discussed above. Raman was not feasible using the employed flow-cell, which due to its dead volume of $1 \mathrm{~mL}$. In other works Raman has been employed successfully for the breakthrough detection of $\operatorname{IgG~[36].~Other~reasons~might~be~the~differing~}$ integration time or the salt gradient overlaying the elution.

\subsection{Proposed Control Strategy including PAT}

The proposed process is a continuous, chromatography-reduced process, as this process offers the most economical production of monoclonal antibodies, as shown in another work [5]. Robust process control is possible using either an advanced process control based on a digital twin, as shown in this work. To enable process control, an inline concentration measurement has to be implemented. As shown in this study, PLSR- 
based spectroscopic methods enable a real-time, accessible process control in all used unit operations. The proposed process is illustrated in Figure 18 to Figure 20. In Figure 17, an overview of the course of purity, yield, titer, and DNA concentration in this study is given. Product titer is mainly increased by chromatography and adjusted by UF/DF. Purity is steadily increasing over the course of the process. In ATPE capture, most of the high molecular weight side components are eliminated. These mainly consist of multi-charged DNA molecules, which stay in the salt-dominated heavy phase [76]. Purification occurs in precipitation as most light molecular weight host cell proteins stay in the supernatant, and are eliminated through filtration of precipitates [29]. After chromatographic polishing (IEX and HIC), over $99 \%$ purity is achieved. HMW, as well as LMW side components, are below the detection limit of the applied analytic technologies.

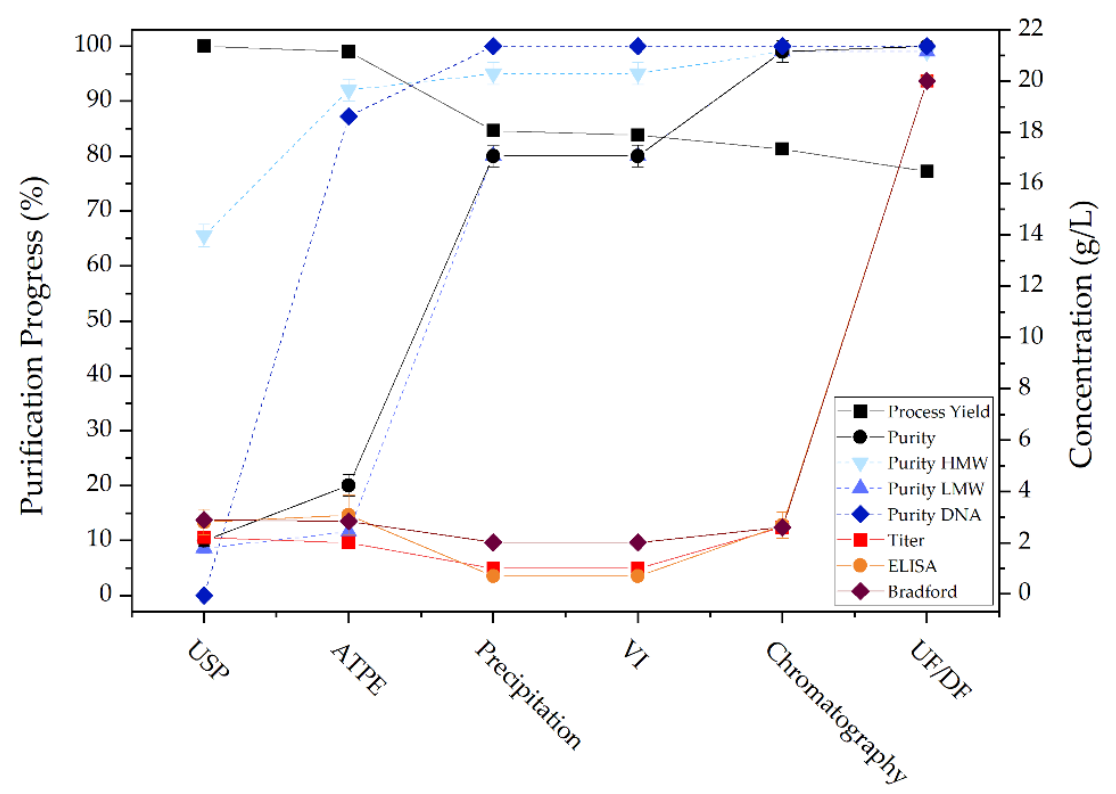

Figure 17. Course of purity, yield, and titer during the process.

As shown in the simulation studies ATPE can sustain a constant $\mathrm{mAb}$ concentration, even when titer in USP fluctuates $+/-50 \%$. This is achieved by calculating the necessary polymer and salt concentration based on the phase equilibrium. As discussed before, Raman is the recommend spectroscopic technique to analyze mAb concentration in USP and ATPE, and is therefore the primary detector at the inlet and product phase outlet stream, as shown in Figure 18.

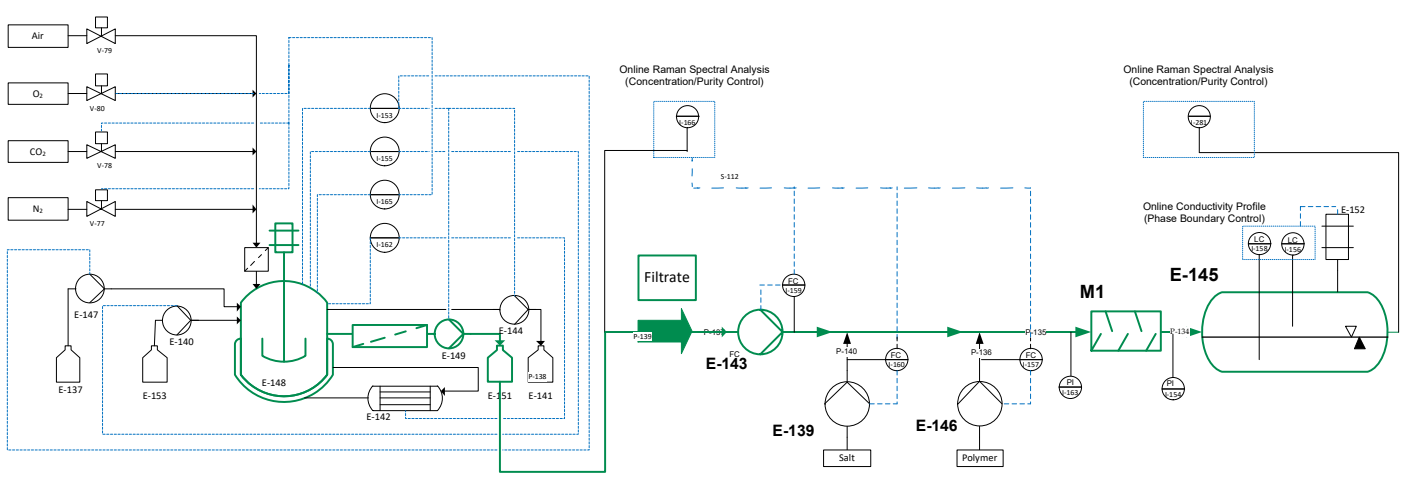

Figure 18. Proposed control strategy for USP and ATPE. A Raman probe is used as (PAT) at the filtrate outlet of USP and forwards $\mathrm{mAb}$ and side component concentrations to the ATPE, which adjusts the polymer and salt concentrations to produce light phase with a constant $\mathrm{mAb}$ concentration which is then forwarded to the precipitation unit. 
Figure 19 shows the process control strategy for the precipitation unit. Precipitation is depicted in a red and dissolution in a green box. The unit consists of four hollow fiber modules that are timed and to enable continuous processing. Phase one is the filtration of precipitates, followed by a washing step in phase two. Redissolution takes place in phase three and finally the module is regenerated by a rinsing process in phase four. After regeneration, the process restarts with the filtration of precipitates. Each module passes through all four phases with a time delay, which is controlled by pressure sensors to ensure the operation range for the hollow fiber membranes. In the dissolution recycling loop, inline measurements and proteomics are installed to detect concentration and purity of the target component.

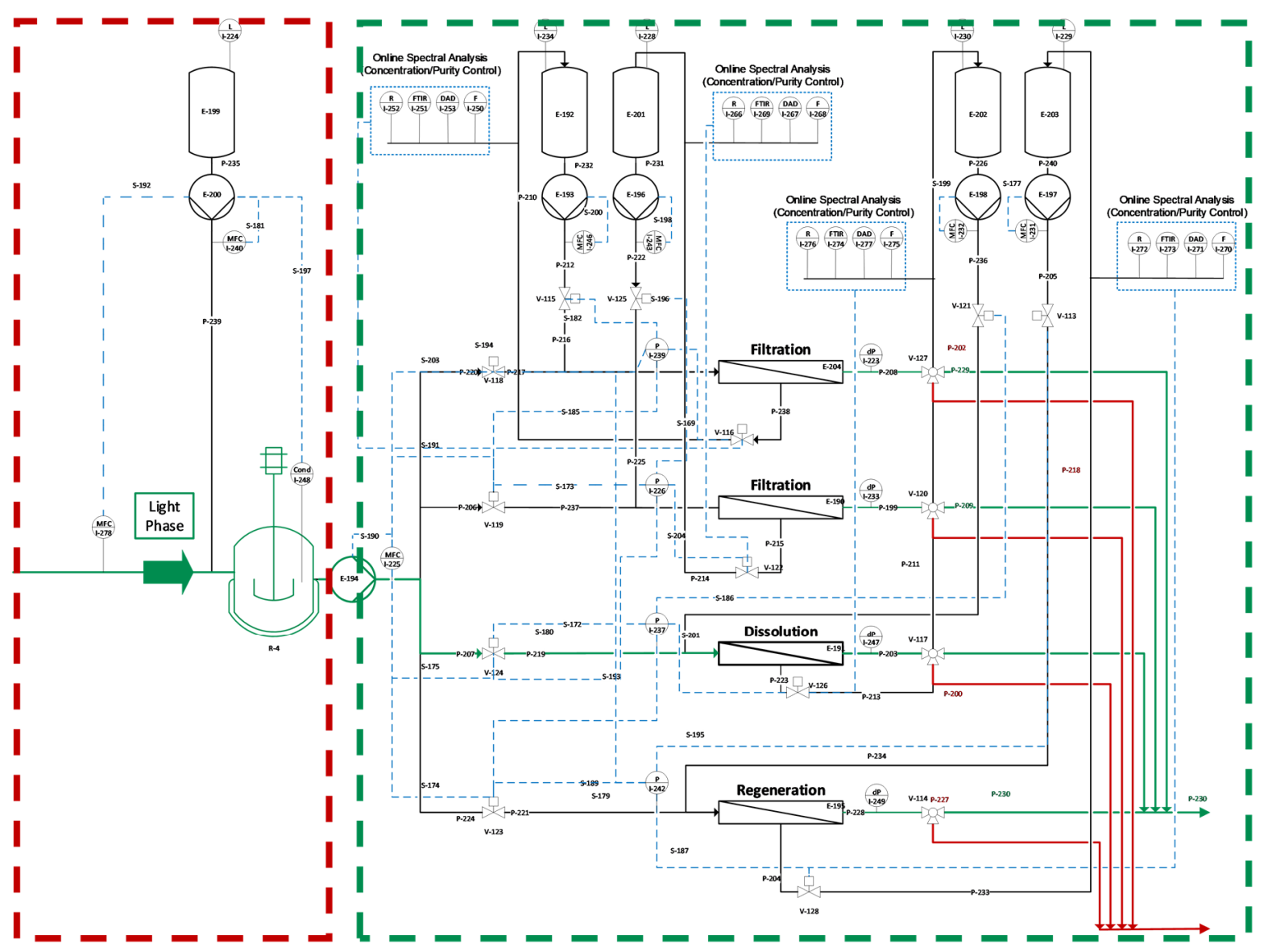

Figure 19. Process control strategy for precipitation. A PAT measurement array at the outlet of the ATPE forwards mAb and side component concentrations to the precipitation unit, which adjusts filtration and dissolution times to obtain a constant $\mathrm{mAb}$ concentration in the dissolution solution.

This measurement technique is used as switching criterion between valves in phase one to abort dissolution as soon as the target component has sufficiently redissolved into the buffer. Finally, the product is filtered through the membrane and transferred to the chromatography step, as well as values of product titer and purity, which are used as model input parameters for chromatography.

Figure 19 shows the flow sheet of the precipitation unit including online measurement trains as well as pressure sensors and mass flow controllers. The unit consists of four membrane modules that are controlled by the digital twin and the integrated inline PAT. Each module passes through different phases with a time delay. In this way continuous processing is enabled. Phase one is the filtration of precipitates, followed by redissolution of the target component. Then, the module is regenerated by a rinsing process. After regeneration, the process restarts with the filtration of precipitates. The mass flow controller 
monitors the incoming flow rate of light phase after ATPE, which is used to calculate the needed precipitant flow rate. Pressure sensors measure the transmembrane pressure during filtration and ensure that the operation range is maintained. Furthermore, a critical pressure is used to switch valve positions and redirect the dispersion to the next filter. The first loaded module passes on to the next phase, the dissolution. Input concentration from ATPE and the predefined final $\mathrm{mAb}$ concentration are used to determine the buffer volume for redissolution of the target component. The inline measurement train in the circuit controls the speed of the dissolution buffer pump as well as the valves to the product tank. As soon as the dissolution of the antibody has reached a stationary value, the dissolution is terminated and the product solution is passed on to the next unit operation.

In Figure 20, a control strategy for iCCC is proposed. Data needed to set the loading time of IEX is either transferred by the previous unit operation or measured in-line in front of the feed tank (blue). Data used for release testing is obtained by measurement arrays located after the columns (green). From the obtained data, two different control strategies for the iCCC unit arise. Firstly, the process can be controlled using the real-time measured concentration as a switch criteria for the fractionation valves. Since the elution order of the components in known, and this order is not subject to change in the process, the product fraction can be cut easily when both light molecular weight side components were eluted. Cutting the product peak at the lowest concentration detectable (i.e., $0.05 \mathrm{~g} / \mathrm{L}$ ) resulted in a yield of over $99 \%$ consistently. Secondly, the iCCC process can be simulated with the help of a digital-twin. Here, further optimizations of space-time yield or purity are possible since the separation can be further optimized. Using the data obtained by the PAT arrays (green), resin aging can be detected or even integrated continuously into the digital twin model, by adjusting model parameters.

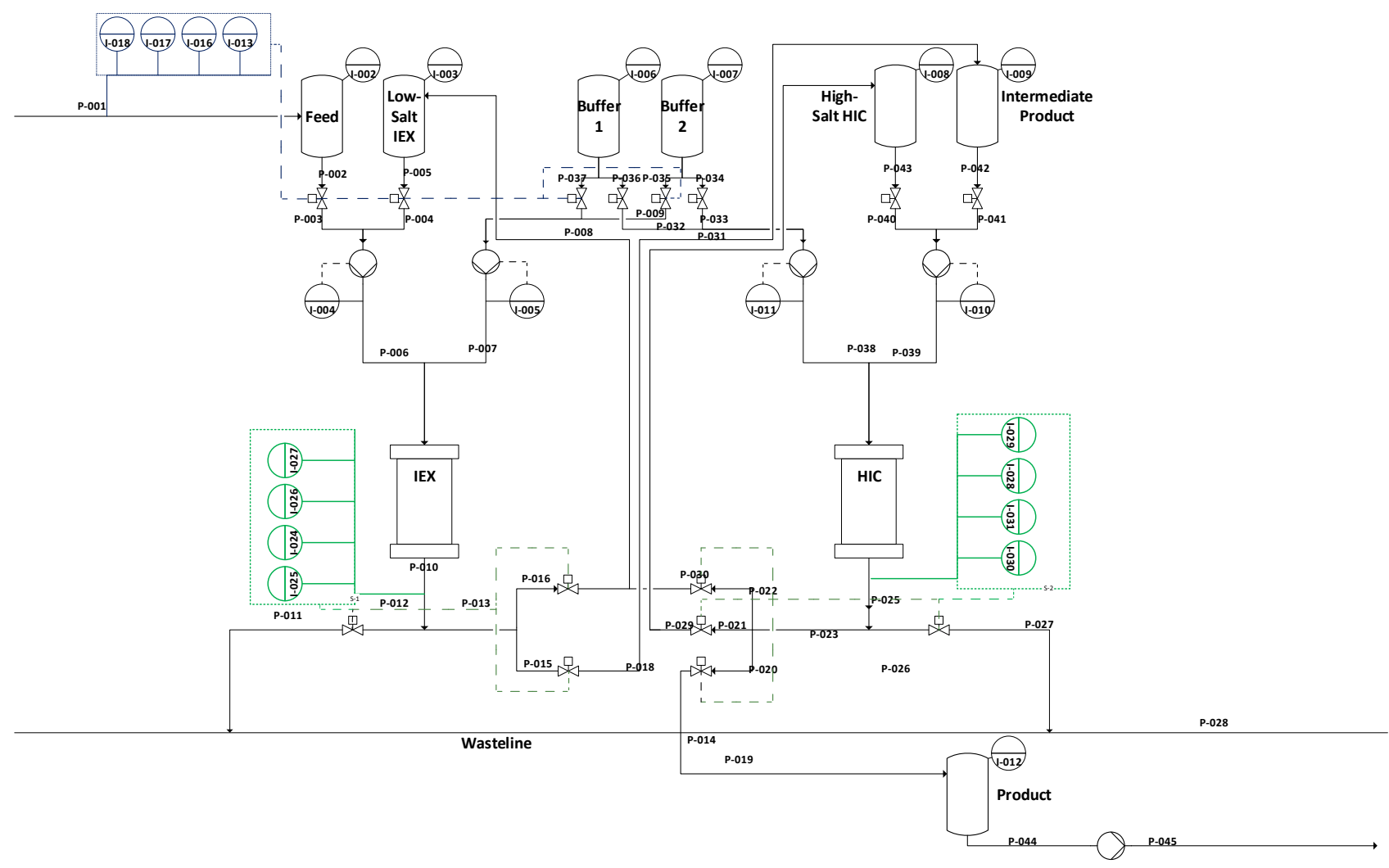

Figure 20. Proposed control strategy for iCCC. PAT measurement information from the precipitation outlet (top left, blue) is used to control the loading time of the IEX. Fractionation in the IEX can also be monitored and controlled by the described PAT detectors (green). 
After chromatography, the mAb concentration is determined by the combination of $\mathrm{DAD}$ and fluorescence. This information determines the necessary concentration factor. Transmembrane pressure is adjusted to achieve the desired concentration. Conductivity measurements are used to determine if the UF/DF operates within the specification limits. Finally, transmembrane pressure for final buffer adjustment towards formulation is adjusted to achieve the desired concentration. Conductivity measurements are used to determine if the UF/DF operates within the specification limits. Lyophilization as the final formulation step before fill and finish has already been described [30].

\section{Conclusions}

In the present study, for a whole mAb manufacturing process, the applicability of a combination of spectroscopic methods has been evaluated to enable APC in continuous manufacturing by PAT.

In USP and the following direct ATPE, Raman-, FTIR-, fluorescence-, and UV/Vis spectroscopy have been successfully applied for titer as well as purity prediction. Raman was the most versatile and robust method and is recommended as primary PAT. In precipitation, similar results were obtained for titer determination. Prediction of purity was challenging for FTIR, fluorescence and UV/Vis, but achievable by Raman spectroscopy. In chromatography, the combination of UV/Vis and fluorescence spectroscopy was able to overcome difficulties in titer and purity prediction induced by overlapping side component spectra, often already reported in literature as well. In final UF/DF, before lyophilization, $\mathrm{UV} /$ Vis spectroscopy is applicable for titer concentration determination, however, for high concentration processes, it is important to ensure the DAD employed is able to operate at elevated concentrations. Raman spectroscopy is especially useful in early stages of the process, whereas more traditional detector technology concepts, such as DAD, can be used in late process stages. The combination of spectroscopic data improves the predictivity as shown for chromatography.

Continuous operation generates much smaller hold-up volumes than batch processing. This causes much shorter start-up und shut-down times with smoother systems responses. In addition, system response is much shorter and smoother, i.e., nearly constant, around the continuous operation point. Hence, detector signal acquisition times and corresponding sampling scan rates for continuous processing are much lower than for comparable batch operation. Changes due to natural system variances are less steep, i.e., continuously near constant system responses. In contrast, only typical gradient elution chromatography operation with fraction cut points at steep chromatogram concentration slopes challenge the accuracy in time resolution. These changes in concentration occur within a few seconds, whereas the feasible number of measuring points is limited by the sampling rate, which leads to a resolution of 10-50 points per peak. This results in an image of the concentration profile that is not sufficiently accurate for fractionation. Only typical breakthrough curves of flow-through operation mode in capture steps differ, and are, as well, sufficiently describable with higher acquisition times and averaged scans.

Based on the developed spectroscopic predictions, dynamic process control of the unit operations was demonstrated for the total process in sophisticated simulation studies based on validated digital twins available for all unit operations. As such, a PAT development workflow for any holistic process development is proposed.

To our knowledge, this comprehensive demonstration of the combination of the applicability of different spectroscopic methods, in the context of a holistic process development, including the complete total process simulation of an APC concept based on digital twins, has been shown for the first time, opening up innovative autonomous operation concepts for the future. The next steps will be the transfer towards different biologic types, such as antibody fragments, peptides, and VLPs available at the institute, as well as piloting studies of the APC conception proposed. 
Author Contributions: This is a joint work by all authors, with each author being especially in charge of his/her expert topic: H.H. for upstream processing, A.S. for liquid-liquid extraction, L.J.L. for precipitation, H.H., C.J., and A.S. for membrane processes, M.M. for process control, A.J. for lyophilization, S.Z.-R. and F.L.V. for chromatography, J.S. acts as the main supervisor. All authors have read and agreed to the published version of the manuscript.

Funding: The authors want to thank the Bundesministerium für Wirtschaft und Energie (BMWi), especially M. Gahr (Projektträger FZ Jülich), for funding the scientific work.

Institutional Review Board Statement: Not applicable.

Informed Consent Statement: Not applicable.

Data Availability Statement: Data can not be made publicly available.

Acknowledgments: The authors would like to thank Reinhard Ditz, formerly of Merck KGaA, Darmstadt, for paper revision and fruitful discussions, as well as the ITVP lab-team, especially Frank Steinhäuser, Volker Strohmeyer and Thomas Knebel, for their efforts and support.

Conflicts of Interest: The authors declare no conflict of interest.

$\begin{array}{ll}\text { Abbreviations } \\ \text { APC } & \text { advanced process control } \\ \text { ATPE } & \text { aqueous two-phase extraction } \\ \text { ATR } & \text { Attenuated total reflectance } \\ \text { DoE } & \text { design of experiments } \\ \text { DSP } & \text { downstream processing } \\ \text { EMA } & \text { European Medicines Agency } \\ \text { FDA } & \text { Food and Drug administration } \\ \text { FTIR } & \text { Fourier-transformed infrared spectroscopy } \\ \text { HIC } & \text { hydrophobic interaction chromatography } \\ \text { iCCC } & \text { integrated counter-current chromatography } \\ \text { IEX } & \text { ion exchange chromatography } \\ \text { IPC } & \text { inline process control analytics } \\ \text { mAb } & \text { monoclonal antibody } \\ \text { MCSGP } & \text { multicolumn countercurrent solvent gradient purification } \\ \text { MPC } & \text { model-based process control } \\ \text { MS } & \text { mass spectrometry } \\ \text { PAT } & \text { process analytical technology } \\ \text { PCA } & \text { principle component analysis } \\ \text { PCS } & \text { process control system } \\ \text { PLSR } & \text { Partial least squares regression } \\ \text { Prot A } & \text { protein A chromatography } \\ \text { QA } & \text { quality assurance } \\ \text { QbD } & \text { quality-by-design } \\ \text { UF/DF } & \text { Ultrafiltration/diafiltration } \\ \text { USP } & \text { upstream processing } \\ \text { VLP } & \text { virus-like particles } \\ \end{array}$

\section{References}

1. Kornecki, M.; Strube, J. Accelerating Biologics Manufacturing by Upstream Process Modelling. Processes 2019, 7, 166. [CrossRef]

2. Subramanian, G. Continuous Biomanufacturing_Innovative Technologies and Methods; Wiley-VCH Verlag GmbH \& Co. KGaA: Weinheim, Germany, 2017; ISBN 9783527699902.

3. Roos, S.Z.; Schmidt, A.; Mestmäcker, F.; Mouellef, M.; Huter, M.; Uhlenbrock, L.; Kornecki, M.; Lohmann, L.; Ditz, R.; Strube, J. Accelerating Biologics Manufacturing by Modeling or: Is Approval under the QbD and PAT Approaches Demanded by Authorities Acceptable Without a Digital-Twin? Processes 2019, 7, 94. [CrossRef]

4. Walther, J.; Godawat, R.; Hwang, C.; Abe, Y.; Sinclair, A.; Konstantinov, K. The business impact of an integrated continuous biomanufacturing platform for recombinant protein production. J. Biotechnol. 2015, 213, 3-12. [CrossRef] [PubMed] 
5. Kornecki, M.; Schmidt, A.; Lohmann, L.; Huter, M.; Mestmäcker, F.; Klepzig, L.; Mouellef, M.; Roosand, S.Z.; Strube, J. Accelerating Biomanufacturing by Modeling of Continuous Bioprocessing-Piloting Case Study of Monoclonal Antibody Manufacturing. Processes 2019, 7, 495. [CrossRef]

6. $\quad$ Feidl, F.; Vogg, S.; Wolf, M.; Podobnik, M.; Ruggeri, C.; Ulmer, N.; Wälchli, R.; Souquet, J.; Broly, H.; Butté, A.; et al. Process-wide control and automation of an integrated continuous manufacturing platform for antibodies. Biotechnol. Bioeng. 2020, 117, 1367-1380 [CrossRef]

7. Roos, S.Z.; Mouellef, M.; Siemers, C.; Strube, J. Process Analytical Approach towards Quality Controlled Process Automation for the Downstream of Protein Mixtures by Inline Concentration Measurements Based on Ultraviolet/Visible Light (UV/VIS) Spectral Analysis. Antibodies 2017, 6, 24. [CrossRef]

8. Roos, S.Z.; Mouellef, M.; Ditz, R.; Strube, J. Distinct and Quantitative Validation Method for Predictive Process Modelling in Preparative Chromatography of Synthetic and Bio-Based Feed Mixtures Following a Quality-by-Design (QbD) Approach. Processes 2019, 7, 580. [CrossRef]

9. Kornecki, M.; Schmidt, A.; Strube, J. Pat as Key-Enabling Technology for Qbd in Pharmaceutical Manufacturing-A Conceptual Review on Upstream and Downstream Processing. Chem. Today 2018, 36, 44-48.

10. Claßen, J.; Aupert, F.; Reardon, K.F.; Solle, D.; Scheper, T. Spectroscopic sensors for in-line bioprocess monitoring in research and pharmaceutical industrial application. Anal. Bioanal. Chem. 2017, 409, 651-666. [CrossRef]

11. Kornecki, M.; Strube, J. Process Analytical Technology for Advanced Process Control in Biologics Manufacturing with the Aid of Macroscopic Kinetic Modeling. Bioengineering 2018, 5, 25. [CrossRef]

12. Santos, R.M.; Kessler, J.-M.; Salou, P.; Menezes, J.C.; Peinado, A. Monitoring mAb cultivations with in-situ raman spectroscopy: The influence of spectral selectivity on calibration models and industrial use as reliable PAT tool. Biotechnol. Prog. 2018, 34, 659-670. [CrossRef] [PubMed]

13. Absi, N.R.A.; Kenty, B.M.; Cuellar, M.E.; Borys, M.C.; Sakhamuri, S.; Strachan, D.J.; Hausladen, M.C.; Li, Z.J. Real time monitoring of multiple parameters in mammalian cell culture bioreactors using an in-line Raman spectroscopy probe. Biotechnol. Bioeng. 2011, 108, 1215-1221. [CrossRef] [PubMed]

14. Suvarov, P.; Wouwer, A.V.; Lee, J.W.; Morgensten, A.S.; Kienle, A. Control of incomplete separation in simulated moving bed chromatographic processes. IFAC-PapersOnLine 2016, 49, 153-158. [CrossRef]

15. Engell, S.; Toumi, A. Optimization and Control of Chromatography. In European Symposium on Computer-Aided Process Engineering14, Proceedings of the 37th European Symposium of the Working Party on Computer-Aided Process Engineering, ESCAPE-14, Lisbon, Portugal, 16-19 May 2004, 1st ed.; Póvoa, A.B., Matos, H., Eds.; Elsevier: Amsterdam, The Netherlands, 2004; pp. 9-22, ISBN 9780444516947.

16. Strube, J.; Klatt, K.U.; Noeth, G.; Greifenberg, J.; Bocker, S.; Kansy, H.; Jahn, P.; Justen, B. Modular Valve System for Countercurrent Chromatography Process. U.S. Patent US8658040B2, 25 February 2014.

17. Godawat, R.; Brower, K.; Jain, S.; Konstantinov, K.; Riske, F.; Warikoo, V. Periodic counter-current chromatography-Design and operational considerations for integrated and continuous purification of proteins. Biotechnol. J. 2012, 7, 1496-1508. [CrossRef] [PubMed]

18. Späth, T.M.; Aumann, L.; Melter, L.; Ströhlein, G.; Morbidelli, M. Chromatographic separation of three monoclonal antibody variants using multicolumn countercurrent solvent gradient purification (MCSGP). Biotechnol. Bioeng. 2008, 100, $1166-1177$. [CrossRef]

19. Strube, J. Technische Chromatographie: Auslegung, Optimierung, Betrieb und Wirtschaftlichkeit; Shaker Verlag GmbH: Düren/Maastricht, Germany, 2000; ISBN 3826568974.

20. Böcker, S.; Greifenberg, J.; Jähn, P.; Justen, B.; Kansy, H.; Klatt, K.U.; Noeth, G.; Strube, J. Process for the Preparation of Chemical and Pharmaceutical Products with Integrated Multi-Col. Available online: umnChromatography.https://patents.google.com/ patent/US7615159B2/en (accessed on 1 December 2020).

21. Brestrich, N.; Briskot, T.; Osberghaus, A.; Hubbuch, J. A tool for selective inline quantification of co-eluting proteins in chromatography using spectral analysis and partial least squares regression. Biotechnol. Bioeng. 2014, 111, 1365-1373. [CrossRef]

22. Buijs, J.; Norde, W.; Lichtenbelt, J.W.T. Changes in the Secondary Structure of Adsorbed IgG and F(ab')2 Studied by FTIR Spectroscopy. Langmuir 1996, 12, 1605-1613. [CrossRef]

23. International Council for Harmonisation of Technical Requirements for Pharmaceuticals for Human Use (ICH). Endorsed Guide for ICH Q8/Q9/Q10 Implementation. 2011. Available online: https://database.ich.org/sites/default/files/Q8_Q9_Q10_Q\%26As_R4 _Points_to_Consider_0.pdf (accessed on 3 December 2020).

24. Wasalathanthri, D.P.; Feroz, H.; Puri, N.; Hung, J.; Lane, G.; Holstein, M.; Chemmalil, L.; Both, D.; Ghose, S.; Ding, J.; et al. Real-time monitoring of quality attributes by in-line Fourier transform infrared spectroscopic sensors at ultrafiltration and diafiltration of bioprocess. Biotechnol. Bioeng. 2020, 117, 3766-3774. [CrossRef]

25. Huter, M.J.; Strube, J. Model-Based Design and Process Optimization of Continuous Single Pass Tangential Flow Filtration Focusing on Continuous Bioprocessing. Processes 2019, 7, 317. [CrossRef]

26. Roth, T.; Uhlenbrock, L.; Strube, J. Distinct and Quantitative Validation for Predictive Process Modelling in Steam Distillation of Caraway Fruits and Lavender Flower Following a Quality-By-Design (QbD) Approach. Processes 2020, 8, 594. [CrossRef]

27. Schmidt, A.; Strube, J. Distinct and Quantitative Validation Method for Predictive Process Modeling with Examples of LiquidLiquid Extraction Processes of Complex Feed Mixtures. Processes 2019, 7, 298. [CrossRef] 
28. Sixt, M.; Uhlenbrock, L.; Strube, J. Toward a Distinct and Quantitative Validation Method for Predictive Process ModellingOn the Example of Solid-Liquid Extraction Processes of Complex Plant Extracts. Processes 2018, 6, 66. [CrossRef]

29. Lohmann, L.J.; Strube, J. Accelerating Biologics Manufacturing by Modeling: Process Integration of Precipitation in mAb Downstream Processing. Processes 2020, 8, 58. [CrossRef]

30. Klepzig, L.S.; Juckers, A.; Knerr, P.; Harms, F.; Strube, J. Digital Twin for Lyophilization by Process Modeling in Manufacturing of Biologics. Processes 2020, 8, 1325. [CrossRef]

31. Uhlenbrock, L.; Jensch, C.; Tegtmeier, M.; Strube, J. Digital Twin for Extraction Process Design and Operation. Processes 2020, 8, 866. [CrossRef]

32. Huter, M.J.; Jensch, C.; Strube, J. Model Validation and Process Design of Continuous Single Pass Tangential Flow Filtration Focusing on Continuous Bioprocessing for High Protein Concentrations. Processes 2019, 7, 781. [CrossRef]

33. Santos, R.M.; Kaiser, P.; Menezes, J.C.; Peinado, A. Improving reliability of Raman spectroscopy for mAb production by upstream processes during bioprocess development stages. Talanta 2019, 199, 396-406. [CrossRef] [PubMed]

34. Popp, J.; Mayerhöfer, T. Micro-Raman Spectroscopy; De Gruyter: Berlin, Germany, 2020; ISBN 9783110515312.

35. Kosa, G.; Shapaval, V.; Kohler, A.; Zimmermann, B. FTIR spectroscopy as a unified method for simultaneous analysis of intra and extracellular metabolites in high-throughput screening of microbial bioprocesses. Microb. Cell Fact. 2017, 16, 195. [CrossRef]

36. Feidl, F.; Garbellini, S.; Vogg, S.; Sokolov, M.; Souquet, J.; Broly, H.; Butté, A.; Morbidelli, M. A new flow cell and chemometric protocol for implementing in-line Raman spectroscopy in chromatography. Biotechnol. Prog. 2019, 35, e2847. [CrossRef]

37. Thakur, G.; Thori, S.; Rathore, A.S. Implementing PAT for single-pass tangential flow ultrafiltration for continuous manufacturing of monoclonal antibodies. J. Membr. Sci. 2020, 613, 118492. [CrossRef]

38. Faix, O. Fourier Transform Infrared Spectroscopy. In Methods in Lignin Chemistry, 1st ed.; Lin, S.Y., Ed.; Springer: Berlin, Germany, 2011; pp. 83-109. ISBN 978-3-642-74067-1.

39. Barth, A. Infrared spectroscopy of proteins. Biochim. Biophys. Acta 2007, 1767, 1073-1101. [CrossRef] [PubMed]

40. Sellick, C.A.; Hansen, R.; Jarvis, R.M.; Maqsood, A.R.; Stephens, G.M.; Dickson, A.J.; Goodacre, R. Rapid monitoring of recombinant antibody production by mammalian cell cultures using fourier transform infrared spectroscopy and chemometrics. Biotechnol. Bioeng. 2010, 106, 432-442. [CrossRef] [PubMed]

41. Oelmeier, S.A.; Effio, C.L.; Hubbuch, J. Alternative separation steps for monoclonal antibody purification: Combination of centrifugal partitioning chromatography and precipitation. J. Chromatogr. A 2013, 1319, 118-126. [CrossRef] [PubMed]

42. Pei, Y.; Wang, J.; Wu, K.; Xuan, X.; Lu, X. Ionic liquid-based aqueous two-phase extraction of selected proteins. Sep. Purif. Technol. 2009, 64, 288-295. [CrossRef]

43. Audet, M.B.; Kazarian, S.G.; Byrne, B. In-column ATR-FTIR spectroscopy to monitor affinity chromatography purification of monoclonal antibodies. Sci. Rep. 2016, 6, 30526. [CrossRef]

44. Barresi, A.A.; Fissore, D. In-Line Product Quality Control of Pharmaceuticals in Freeze-Drying Processes. In Modern Drying Technology; Tsotsas, E., Mujumdar, A.S., Eds.; Wiley-VCH Verlag GmbH \& Co. KGaA: Weinheim, Germany, 2011 ; pp. 91-154. ISBN 9783527631667.

45. Fissore, D.; Pisano, R.; Barresi, A.A. Process analytical technology for monitoring pharmaceuticals freeze-drying-A comprehensive review. Dry. Technol. 2018, 36, 1839-1865. [CrossRef]

46. Johnson, R.E.; Teagarden, D.L.; Lewis, L.M.; Gieseler, H. Analytical accessories for formulation and process development in freeze-drying. Am. Pharm. Rev. 2009, 12, 54-60.

47. Nail, S.; Tchessalov, S.; Shalaev, E.; Ganguly, A.; Renzi, E.; Dimarco, F.; Wegiel, L.; Ferris, S.; Kessler, W.; Pikal, M.; et al. Recommended Best Practices for Process Monitoring Instrumentation in Pharmaceutical Freeze Drying-2017. AAPS PharmSciTech 2017, 18, 2379-2393. [CrossRef]

48. Patel, S.M.; Pikal, M. Process analytical technologies (PAT) in freeze-drying of parenteral products. Pharm. Dev. Technol. 2009, 14, 567-587. [CrossRef]

49. Read, E.K.; Shah, R.B.; Riley, B.S.; Park, J.T.; Brorson, K.A.; Rathore, A.S. Process analytical technology (PAT) for biopharmaceutical products: Part II. Concepts and applications. Biotechnol. Bioeng. 2010, 105, 285-295. [CrossRef]

50. Schneid, S. Investigation of Novel Process Analytical Technology (PAT) Tools for Use in Freeze-Drying Processes. Ph.D. Thesis, Friedrich-Alexander-Universität Erlangen-Nürnberg, Erlangen, Germany, 2010.

51. Yadav, L.D.S. Ultraviolet (UV) and Visible Spectroscopy; Springer: Amsterdam, The Netherlands, 2005. [CrossRef]

52. Lakowicz, J.R. (Ed.) Introduction to Fluorescence. In Principles of Fluorescence Spectroscopy, 3rd ed.; Springer: New York, NY, USA, 2010; pp. 1-26. ISBN 978-0-387-31278-1.

53. Kelly, C.M.; Welsh, J.; Rodger, A.; Dafforn, T.R.; Thomas, O.R.T. Automated High-Throughput Capillary Circular Dichroism and Intrinsic Fluorescence Spectroscopy for Rapid Determination of Protein Structure. Anal. Chem. 2019, 91, 13794-13802. [CrossRef] [PubMed]

54. Rolinger, L.; Rüdt, M.; Diehm, J.; Chow-Hubbertz, J.; Heitmann, M.; Schleper, S.; Hubbuch, J. Multi-attribute PAT for UF/DF of Proteins-Monitoring Concentration, particle sizes, and Buffer Exchange. Anal. Bioanal. Chem. 2020, 412, 2123-2136. [CrossRef]

55. Jungbauer, A. Real-Time Monitoring of Product Purification. Patent Publication Number WO/2017/174580, 12 October 2017.

56. Barresi, A.A.; Velardi, S.A.; Pisano, R.; Rasetto, V.; Vallan, A.; Galan, M. In-line control of the lyophilization process. A gentle PAT approach using software sensors. Int. J. Refrig. 2009, 32, 1003-1014. [CrossRef] 
57. Bosca, S.; Barresi, A.A.; Fissore, D. Fast freeze-drying cycle design and optimization using a PAT based on the measurement of product temperature. Eur. J. Pharm. Biopharm. 2013, 85, 253-262. [CrossRef]

58. Fissore, D.; Pisano, R. Computer-Aided Framework for the Design of Freeze-Drying Cycles: Optimization of the Operating Conditions of the Primary Drying Stage. Processes 2015, 3, 406-421. [CrossRef]

59. Pisano, R.; Fissore, D.; Barresi, A.A. In-Line and Off-Line Optimization of Freeze-Drying Cycles for Pharmaceutical Products Dry. Technol. 2013, 31, 905-919. [CrossRef]

60. Tang, X.C.; Nail, S.L.; Pikal, M.J. Freeze-drying process design by manometric temperature measurement: Design of a smart freeze-dryer. Pharm. Res. 2005, 22, 685-700. [CrossRef] [PubMed]

61. Buckley, K.; Ryder, A.G. Applications of Raman Spectroscopy in Biopharmaceutical Manufacturing: A Short Review. Appl. Spectrosc. 2017, 71, 1085-1116. [CrossRef] [PubMed]

62. Sauer, D.G.; Melcher, M.; Mosor, M.; Walch, N.; Berkemeyer, M.; Scharl-Hirsch, T.; Leisch, F.; Jungbauer, A.; Dürauer, A. Real-time monitoring and model-based prediction of purity and quantity during a chromatographic capture of fibroblast growth factor 2. Biotechnol. Bioeng. 2019, 116, 1999-2009. [CrossRef]

63. Dziadosz, M.; Lessig, R.; Bartels, H. HPLC-DAD protein kinase inhibitor analysis in human serum. J. Chromatogr. B Analyt. Technol. BioMed Life Sci. 2012, 893-894, 77-81. [CrossRef]

64. JASCO Deutschland GmbH. FP-2020 Fluorescence Detector. Available online: https://www.jasco.de/en/content/FP-2020/ \{\} tpl.index/.html (accessed on 3 December 2020).

65. Park, J.; Nagapudi, K.; Vergara, C.; Ramachander, R.; Laurence, J.S.; Krishnan, S. Effect of pH and excipients on structure, dynamics, and long-term stability of a model IgG1 monoclonal antibody upon freeze-drying. Pharm. Res. 2013, 30, 968-984. [CrossRef] [PubMed]

66. Dittmar, R. Advanced Process. Control.: PID-Basisregelungen, Vermaschte Regelungsstrukturen, Softsensoren, Model. Predictive Control; De Gruyter: Berlin, Germany, 2017; ISBN 978-3-11-049997-1.

67. Keerthi, S.S.; Gilbert, E.G. Optimal infinite-horizon feedback laws for a general class of constrained discrete-time systems: Stability and moving-horizon approximations. J. Optim. Theory Appl. 1988, 57, 265-293. [CrossRef]

68. Song, I.-H.; Amanullah, M.; Erdem, G.; Mazzotti, M.; Rhee, H.-K. Experimental implementation of identification-based optimizing control of a simulated moving bed process. J. Chromatogr. A 2006, 1113, 60-73. [CrossRef] [PubMed]

69. Sommeregger, W.; Sissolak, B.; Kandra, K.; von Stosch, M.; Mayer, M.; Striedner, G. Quality by control: Towards model predictive control of mammalian cell culture bioprocesses. Biotechnol. J. 2017, 12. [CrossRef] [PubMed]

70. Fanali, S. Editorial on "Simulated moving bed chromatography for the separation of enantiomers" by A. Rajendran, G. Paredes and M. Mazzotti. J. Chromatogr. A 2009, 1216, 708. [CrossRef] [PubMed]

71. Diederich, P.; Hansen, S.K.; Oelmeier, S.A.; Stolzenberger, B.; Hubbuch, J. A sub-two minutes method for monoclonal antibodyaggregate quantification using parallel interlaced size exclusion high performance liquid chromatography. J. Chromatogr. A 2011, 1218, 9010-9018. [CrossRef]

72. Rüdt, M.; Andris, S.; Schiemer, R.; Hubbuch, J. Factorization of preparative protein chromatograms with hard-constraint multivariate curve resolution and second-derivative pretreatment. J. Chromatogr. A 2019, 1585, 152-160. [CrossRef] [PubMed]

73. Martins, D.L.; Sencar, J.; Hammerschmidt, N.; Flicker, A.; Kindermann, J.; Kreil, T.R.; Jungbauer, A. Truly continuous low pH viral inactivation for biopharmaceutical process integration. Biotechnol. Bioeng. 2020, 117, 1406-1417. [CrossRef]

74. Esbensen, K.H.; Swarbrick, B.; Westad, F.; Whitcombe, P.; Andersen, M. Multivariate Data Analysis. In An Introduction to Multivariate Analysis, Process Analytical Technology and Quality by Design, 6th ed.; CAMO Software AS.: Magnolia, TX, USA, 2018; ISBN 978-82-691104-0-1.

75. Helling, C.; Dams, T.; Gerwat, B.; Belousov, A.; Strube, J. Physical characterization of column chromatography: Stringent control over equipment performance in biopharmaceutical production. Trends Chromatogr. 2013, 8, 55-71.

76. Kruse, T.; Kampmann, M.; Greller, G. Aqueous Two-Phase Extraction of Monoclonal Antibodies from High Cell Density Cell Culture. Chem. Ing. Tech. 2020, 256, 41. [CrossRef] 